This document is the Accepted Manuscript version of a Published Work that appeared in final form in The Journal of Physical Chemistry C, copyright (C) American Chemical Society after peer review and technical editing by the publisher. To access the final edited and published work see https://pubs.acs.org/articlesonrequest/AOR-EJukdSe7YZM2sYuihTrG.

\title{
Distinguishing between Charge Transfer Mechanisms at Organic/Inorganic Interfaces Employing Hybrid Functionals
}

Elisabeth Wruss, Egbert Zojer and Oliver T. Hofmann*

Institute of Solid State Physics, NAWI Graz, Graz University of Technology, 8010 Graz, Austria.

\section{Corresponding Author}

*o.hofmann@tugraz.at 


\section{Abstract}

When modeling inorganic-organic interfaces with density functional theory, the outcome often depends on the chosen functional. Hybrid functionals, which employ a fraction of Hartree-Fock exchange $\alpha$, tend to give better results than the more commonly applied semilocal functionals, since they remove or at least mitigate the unphysical electron selfinteraction. However, the choice of $\alpha$ is not straightforward, as its effect on observables depends on the physical properties of the investigated system, such as the size of the investigated molecule, and the polarizability of the substrate. In this contribution, we demonstrate this impact exemplarily for tetrafluoro-1,4-benzoquinone TFBQ on semiconducting (copper-I-oxide $\mathrm{Cu}_{2} \mathrm{O}$ ) and metallic $(\mathrm{Cu})$ substrates and explore how the simulated charge transfer depends on $\alpha$. We determine the value $\alpha^{*}$ that marks the transition point between spurious over-localization and over-delocalization of charges. This allows us to shed light on the interplay between the value of $\alpha^{*}$ and the physical properties of the interface. We find that on the inert, semiconducting substrate $\alpha^{*}$ strongly depends on surface screening. Furthermore, $\alpha$ has a significant impact on the charge transfer, and, in particular, the charge localization. Conversely, for the adsorption on $\mathrm{Cu}, \alpha$ affects only the amount of transferred charge, but not its localization, which is a consequence of strong hybridization. Finally, we discuss limitations to the predictive power of DFT for modeling charge transfer at hybrid interfaces and explain why the choice of a "correct" amount of Hartree-Fock exchange is difficult, if not impossible. However, we argue why simulations still provide valuable insights into the charge transfer mechanism at organic/inorganic interfaces and describe how $\alpha$ can be chosen sensibly to simulate any given system. 


\section{Introduction}

With increasing computational power available to scientific applications, computer simulations have become more and more significant in various scientific disciplines. A showcase field concerning this trend is the investigation of inorganic/organic interfaces. As they are often embedded within the investigated specimen, experimental investigations can be troublesome. Moreover, experiments frequently yield only incomplete information and do not provide a full atomistic understanding of the processes relevant at interfaces. Here, firstprinciple simulations can provide complementary insights and potentially offer an entirely different perspective by making observables accessible which are difficult to track experimentally.

The most widely used simulation method in material science based on the quantummechanical description of matter is Density Functional Theory (DFT). The reason for this is mainly that DFT offers a good compromise between computational efficiency and accuracy. ${ }^{1}$ While DFT is in principle exact, in practice approximations need to be made, since the exact functional is not known. A large variety of different exchange-correlation functionals is available and many basic properties, such as covalent bonds, are well described within most of the existing frameworks. ${ }^{2-4}$

Unfortunately, this is not necessarily true for all observables, and inconsistencies between different functionals occur. In particular when charge transfer at interfaces come into play, the results of different DFT functionals can differ, varying both the amount of transferred charge and the charge distribution within a given material combination. ${ }^{5-11}$ The problem of spurious charge transfer is particularly pronounced at inorganic/organic interfaces, where two very different classes of materials are combined. ${ }^{10}$ Conceptually, for such interfaces, two 
fundamentally different charge transfer mechanisms have been found both in simulations and in experiments: Fractional Charge Transfer (FCT) and Integer Charge Transfer (ICT). ${ }^{12}$ FCT implies that all molecules get equally fractionally charged. It occurs on surfaces where hybrid bands between substrate and molecules can be formed, which is, for example, the case for interfaces with pristine metals. ${ }^{13-16}$ The case of ICT is typically found for less reactive surfaces, such as passivated metals or semiconductors. ${ }^{17-21}$ There, charge transfer can occur only in integer electron numbers due to the absence of hybrid bands. This leads to the coexistence of charged and neutral molecules on the surface. ${ }^{12,20,22}$ The difference between ICT and FCT, therefore, lies primarily in the localization of charge within the molecular adsorbate. ${ }^{12}$ Consequently, the charge transfer mechanism strongly influences the electrostatic landscape and, therefore, several physical properties of the interface ${ }^{20}$ (such as charge injection barriers $\left.{ }^{23}\right)$. In this work, we address the question how well the typically applied semi-local and hybrid DFT methods are suited to do reliably describe the properties of such hybrid interfaces.

A complication that arises when trying to distinguish between ICT and FCT in simulations is that semi-local DFT functionals (such as PBE, ${ }^{24,25}$ which is also applied in this work) spuriously over-delocalize charge. ${ }^{8,26-29}$ Consequently, by default FCT is favored. The problem of overdelocalization can be overcome by admixing a fraction of Hartree-Fock exchange (quantified by the parameter $\alpha$ ). This is done in so-called hybrid functionals. ${ }^{30}$ It has been argued that Hartree-Fock exchange plays a similar role as the on-site repulsion term in the Hubbard model (i.e. $+U$ term), ${ }^{31-33}$ and it thus relates to the difference between the first and the second ionization energy, which is naturally system-dependent.

While a too low fraction of Hartree-Fock exchange favors FCT, conversely, when a (too) high amount of Hartree-Fock exchange is chosen, this leads to the opposite case of charge over- 
localization ${ }^{11,34}$ and causes an unphysical favoring of ICT over FCT. ${ }^{20}$ Therefore, the charge transfer mechanism obtained in a simulation can depend strongly on the applied functional and the simulation does not necessarily reflect the correct physics.

In this contribution, we address several questions concerning the interplay between the chosen functional and the charge transfer properties obtained when simulating hybrid interfaces. In particular, we investigate whether semi-local and hybrid functionals can predict the charge transfer mechanism. In addition, we discuss how insight can still be gained in systems where this is not the case. In this context, we also consider the question, how well typically applied "default" hybrid functionals, such as PBEO (which employs $\alpha=0.25$ ), $3,30,35$ are able to describe charge transfer at interfaces.

We start by reviewing the physics behind the two charge transfer mechanisms and how they are represented within DFT. Readers who are more interested in the practical application are encouraged to continue with section 4 , where we simulate different interfaces and investigate the influence of $\alpha$. Throughout, we apply the PBEh ${ }^{30}$ functional family. We investigate two prototypical interfaces which are expected to exhibit different charge transfer mechanisms. These comprise the adsorption of tetrafluoro-1,4-benzoquinone (TFBQ, see Figure 1) on a weakly reactive, metallic surface and on an essentially inert semiconducting substrate, where (almost) no hybridization between adsorbate and substrate takes place.

TFBQ is an electron acceptor and, therefore, triggers electron transfer processes on substrates with a sufficiently small work function. Initially, we analyze the molecule in the gas phase to obtain a starting point for our discussion. We then consider adsorption on the semiconducting copper-I-oxide $\mathrm{Cu}_{2} \mathrm{O}$ surface. In case of intrinsic $\mathrm{Cu}_{2} \mathrm{O}$, the LUMO of TFBQ lies in the gap of the substrate, and no charge transfer toward the molecule takes place. To obtain 
a prototypical ICT scenario, charge transfer is enabled by inducing an $n$-doped situation of the substrate, which results in filling of the molecular LUMO. Then, we simulate adsorption on $\mathrm{Cu}$, where we expect that the hybridization between molecule and substrate will lead to an FCT situation.

For the discussed systems, we derive the transition value $\alpha^{*}$, which is defined such that the applied functional does neither over-localize nor over-delocalize charge. Comparing this value for physically different systems allows us to investigate the interplay of physical properties and the applied functional. We discuss why it is problematic to choose an "ideal" value of $\alpha$ for a given system and how to most reasonably apply DFT for the investigation of different charge transfer mechanisms.

\section{Methodology}

\subsection{Computational Settings}

All calculations are performed within the FHI-aims simulation package. ${ }^{36}$ We apply the $\mathrm{PBEh}^{30}$ hybrid functional family, which connects PBE and Hartree-Fock exchange according to the equation

$$
E_{x C}^{P B E h}=\alpha E_{x}^{H F}+(1-\alpha) E_{x}^{P B E}+E_{c}^{P B E}
$$

with the exchange-correlation energy $E_{x c}^{P B E h}$ consisting of a portion of Hartree-Fock exchange energy $E_{x}^{H F}$, the complementary portion of PBE exchange $E_{x}^{P B E}$, and the correlation energy $E_{c}^{P B E}$. The relative weight of exchange from PBE and Hartree-Fock is determined by the mixing parameter $\alpha$. The PBEh family contains two commonly used functionals, $\operatorname{PBE}^{24,25}(\alpha=0)$, and PBEO $^{3,30,35}(\alpha=0.25)$. To demonstrate the impact of the fraction of Hartree-Fock exchange, in this work we vary $\alpha$ between 0 and 1 . 
Geometry optimizations are performed for all discussed systems with the corresponding functional. In these optimizations, the vdW-TS van der Waals correction ${ }^{37}$ is applied and for adsorption on $\mathrm{Cu}(111)$ the surface parameterization by Ruiz et al. ${ }^{38}$ is used. We allow the molecule(s) as well as the topmost part of the substrate (i.e., the first O-Cu-O triple layer for $\mathrm{Cu}_{2} \mathrm{O}$ and the two topmost layers for $\mathrm{Cu}$ ) to relax until remaining forces below $0.01 \mathrm{eV} / \AA$ are reached. Full geometry optimizations for every applied functional turns out to be crucial, as incorrect results are obtained for several observables when hybrid-functional calculations on the basis of PBE geometries are performed: For the systems discussed here, hybrid calculations on the basis of PBE geometries result in work-function differences of up to 0.4 $\mathrm{eV}$, adsorption height modifications of up to $0.25 \AA$ and the appearance of spurious peaks in the density of states (DOS) compared to consistently applying the hybrid functional (for details see the Supporting Information).

Note that the performed hybrid geometry optimizations for surface systems containing several hundred atoms are computationally extremely costly. This is aggravated by the need for performing all calculations in a spin-polarized manner, as this is a pre-requisite for capturing the ICT scenario (see below).

For the smallest possible $\mathrm{Cu}_{2} \mathrm{O}(111)$ surface unit cell containing four $\mathrm{Cu}$ atoms (and two $\mathrm{O}$ atoms) per layer, a k-point grid of $12 \times 12 \times 1$ k-points is applied. For metallic $\mathrm{Cu}$, a denser k-grid is required and we used $16 \times 16 \times 1$ k-points for the equivalent $\mathrm{Cu}(111)$ surface unit cell with four atoms per layer. For the larger unit cells containing adsorbed molecules, the k-point grids are scaled accordingly. A Gaussian occupation scheme with a broadening of $0.1 \mathrm{eV}$ is applied.

FHI-aims contains predefined collections of settings for atomic species regarding basis sets, integration grids, and the numerical accuracy of the Hartree potential. ${ }^{36}$ During our 
convergence tests, "light" settings showed sufficient accuracy for the investigated systems when describing observables like the density of states, orbital energies and work functions. Details on the convergence tests are contained in the Supporting Information.

\subsection{Investigated Systems and Unit Cells}

For the surface calculations presented in this work, we consider unit cells containing two molecules of TFBQ on the stable copper-I-oxide $\mathrm{Cu}_{2} \mathrm{O}(111)^{39,40}$ and copper $\mathrm{Cu}(111)$ surfaces (as chemically related examples for semiconductor and metal substrates).

Note that the simulation of several molecules in one unit cell is necessary to describe the coexistence of charged and uncharged molecules as it can appear in the ICT case.

By this material choice, similar binding properties for the molecule are preserved, as on both substrates molecular adsorption is guided by the bonding of the molecular $\mathrm{O}$ atoms to substrate $\mathrm{Cu}$ atoms. We have chosen the mixed-terminated (111)-surface because there the TFBQ molecule exhibits only very weak hybridization with this substrate surface. We note that stronger hybridization between the adsorbate and a semiconductor can occur, e.g., for the Cu-terminated surface (in which case a FCT solution on a semiconducting substrate could theoretically appear).

In hybrid DFT, the band gap of a material (and the level alignment with an adsorbate) depends on the employed functional. ${ }^{41,42}$ In our calculations, the gap of $\mathrm{Cu}_{2} \mathrm{O}$ varies between nonexistent (metallic, $\alpha=0)$ and $6.8 \mathrm{eV}(\alpha=0.75)$, compared to an experimental band gap of 2.1 $\mathrm{eV} .{ }^{43}$ However, this is less of a concern for the present study, since the exact value of the band gap is not relevant as long as a consistent ordering of substrate and adsorbate states (molecular LUMO in the substrate band gap) is maintained. Indeed, for all applied hybrid functionals $(\alpha>0)$, intrinsic $\mathrm{Cu}_{2} \mathrm{O}$ is correctly described as a semiconductor with the TFBQ 
LUMO lying in its band gap. As a consequence, there is no electron transfer to the molecules. The situation is different when $\alpha=0$ (or very close to 0 ). In that case, the semiconducting nature of the substrate is not captured and $\mathrm{Cu}_{2} \mathrm{O}$ exhibits no band gap. In that case the molecular LUMO is below the substrate Fermi-energy, and charge is spuriously transferred toward the TFBQ molecules. Therefore, the energies for the unoccupied LUMO could not be derived with $\alpha=0$, and corresponding results could not be used in the following, whenever LUMO energies were required for determining $\alpha^{*}$.

The applied coverage is motivated by the structure of the $\mathrm{Cu}_{2} \mathrm{O}(111)$ surface, as the $4 \times 4$ cell (containing $16 \mathrm{Cu}$ atoms per layer) is the smallest possible unit cell allowing the commensurate adsorption of TFBQ in a flat-lying geometry. We, therefore, apply an $8 \times 4$ unit cell for both $\mathrm{Cu}$ and $\mathrm{Cu}_{2} \mathrm{O}$ substrates, which allows the adsorption of two molecules per unit cell in symmetry equivalent positions (see Figure 1 ).

To model the Cu substrate, 4 layers of metal atoms are used. At this slab thickness, the metal work-function is converged to within $0.1 \mathrm{eV}$. To simulate the $\mathrm{Cu}_{2} \mathrm{O}(111)$ surface, three O-Cu$\mathrm{O}$ layers of $\mathrm{Cu}_{2} \mathrm{O}(111)$ are employed, corresponding to a thin film of $\mathrm{Cu}_{2} \mathrm{O}$. This results in a slightly smaller band gap than in a bulk $\mathrm{Cu}_{2} \mathrm{O}$ substrate, but does not affect the situation qualitatively, as in all cases the molecular LUMO is located inside the $\mathrm{Cu}_{2} \mathrm{O}$ gap. The lattice constants are set to $3.63 \AA$ for $\mathrm{Cu}$ and to $4.27 \AA$ for $\mathrm{Cu}_{2} \mathrm{O}$.

Note that in principle, the lattice constant depends on the applied functional. However, we carefully checked that using a fixed value does not affect the results in this work, which is discussed in detail in the Supporting Information.

As periodic boundary conditions in all three spatial directions are applied, a vacuum gap of at least $20 \AA$ is inserted between the periodic replicas of the slabs in the direction perpendicular 
to the surface. In addition, a dipole correction ${ }^{44}$ is used to electrostatically decouple the periodic replicas. 


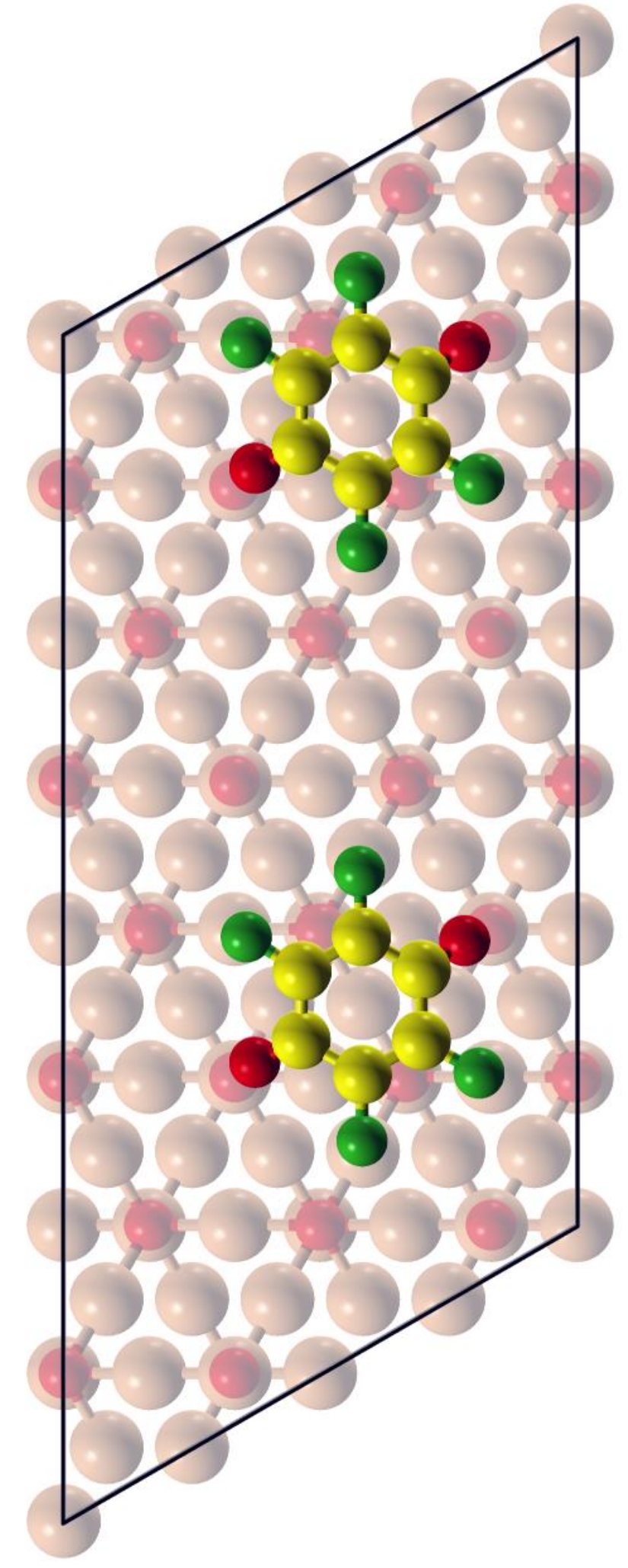

Figure 1: TFBQ on the $\mathrm{Cu}_{2} \mathrm{O}(111)$ surface in the $8 \times 4$ surface supercell. $\mathrm{Cu}$ atoms are depicted in brown, $\mathrm{O}$ atoms in red, $\mathrm{C}$ atoms in yellow, and $\mathrm{F}$ atoms in green. 


\section{Theoretical Background: Modeling Charge Transfer with DFT}

\subsection{Charge Transfer Mechanisms}

A key aspect of the present paper is to understand how the applied DFT methodology influences the charge transfer mechanism found in simulations. Prior to discussing that, we review the physical aspects that lead to the two possible scenarios, fractional charge transfer FCT and integer charge transfer ICT, and highlight the distinguishing features.

Whether FCT or ICT appear in a specific case depends mainly on the hybridization between electronic states of the adsorbed molecules and states of the underlying substrate. Also, the coupling between adjacent molecules can play a role. ${ }^{12,22,45-47}$ To illustrate the two different situations, we consider a model system where all molecules in the layer are notionally equivalent (i.e., they adsorb on equivalent adsorption sites) and where the interaction between the molecules is negligible. This is a good approximation for flat-lying molecules, where the coupling in the first monolayer is typically very small.

At first we discuss a situation where no strong hybridization between the adsorbed molecules and substrate takes place. This is usually the case when there are spacer groups on the molecule separating it from the substrate ${ }^{47}$ or when the molecules are adsorbed on a nonreactive, passivated, or semiconducting substrate. ${ }^{19-21,48-50}$ When the adsorbate is electronically decoupled from the surface, charge transfer is limited to integer electron numbers (e.g., via tunneling). The (spin-)orbitals of the adsorbed molecules are then either completely filled or empty. ${ }^{12}$ As soon as one electron is added to the lowest unoccupied molecular orbital (LUMO) of an adsorbed molecule, the orbital splits into a singly occupied (SOMO) and a singly unoccupied (SUMO) spin orbital (see Figure 2)..$^{20,51,21}$ In the corresponding DOS, the resulting spin-split peaks are located above and below the Fermi level, respectively, while no molecular DOS prevails directly at the Fermi edge. In addition, ICT 
systems exhibit a coexistence of charged and neutral molecules on the surface (where the ratio between the species can adopt a wide range of values).

This breaking of the translational periodicity due to charging can be understood in the following way: Electron transfer is originally triggered by the electron affinities of the molecules in the adsorbate layer being larger than the work function of the substrate (or their ionization energies being smaller). Due to the ensuing transfer of charge to one molecule, the electrostatic energy in its surrounding changes such that further transfer to neighboring molecules is suppressed and neighboring molecules, thus, remain neutral. ${ }^{20}$ For identical adsorption sites and at low temperatures, this results in the formation of a super-lattice of charged molecules. Notably, the charged and neutral molecules not only exhibit differences in their charge densities and densities of states, but also in their geometries (i.e., bond lengths).

Conversely, for adsorption on moderately reactive surfaces (such as clean coinage metals), strong hybridization between the electronic states of substrate and adsorbed molecules can take place and a hybrid band is formed. The states in this hybrid band are delocalized between substrate and molecules. When a state in such a band becomes occupied, part of the associated charge density can be associated with the substrate, and part of it with the adsorbate. This leads to a situation where all molecules on the surface become equally, and often fractionally, charged..$^{12}$ In case of fractional charging, the Fermi-energy cuts through the hybrid-band, i.e., the molecular DOS is non-zero at the Fermi-edge and the net charge on each molecule adopts a non-integer number (see Figure 2). In contrast to the ICT situation, the translational symmetry is not broken and all molecules in the layer exhibit equivalent geometries and electronic structures. The main features distinguishing FCT and ICT systems are summarized in Table 1 and Figure 2. 
Table 1: Characteristics of Integer and Fractional Charge Transfer

\begin{tabular}{|l|l|}
\hline Fractional Charge Transfer & Integer Charge Transfer \\
\hline no spin splitting appears in molecular & spin splitting appears in molecular \\
\hline no breaking of translation symmetry in & symmetry-breaking in molecular layer \\
molecular layer & due to differently charged molecules; \\
\hline all molecules get equally (often & visible in geometry and DOS \\
fractionally) charged & coexistence of charged and neutral \\
\hline non-vanishing DOS at Fermi edge & molecules \\
\hline
\end{tabular}




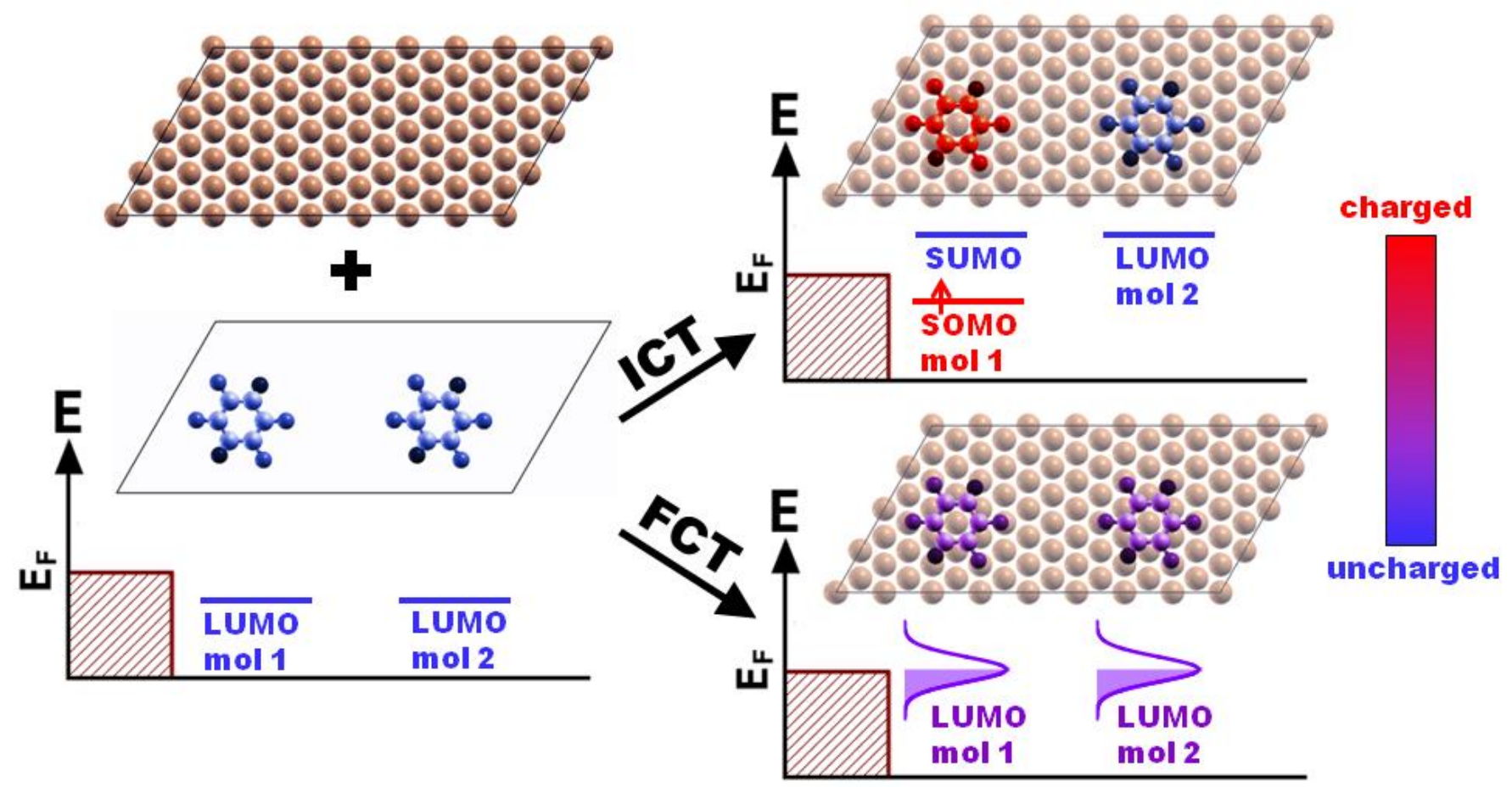

Figure 2: Schematic representation of level alignment upon adsorption of two TFBQ molecules on a substrate. Depending on the interaction, either spin polarized charging of one molecule (ICT) or spin unploarized, equivalent charging of both molecules appears.

\subsection{Impact of the Methodology on the Charge Distribution}

While there is a vast amount of DFT studies describing FCT systems, theoretical work on ICT systems is comparably scarce. This discrepancy appears because modeling ICT is fundamentally more complicated ${ }^{20,22}$ and also computationally significantly more costly than simulating FCT for the following reasons:

The first complication when modeling ICT arises from the periodic boundary conditions, which are required to simulate extended surfaces. To be able to capture symmetry breaking between different molecules in this framework, large supercells containing at least two molecules are required. This already significantly increases the computational effort. Additionally, spin-unrestricted simulations need to be performed, as single (unpaired) 
electrons are transferred. Although this is in principle straightforward to do within most DFT codes, it further increases the computational cost.

The most challenging and computationally most expensive factor is related to the functional required to correctly describe ICT. While FCT can be modeled using semi-local functionals, capturing ICT requires higher-rung methods (such as hybrid functionals). ${ }^{20}$ These are computationally up to two orders of magnitude more demanding than their semi-local counterparts. ${ }^{30}$

We note that the simulation of cells with two molecules per supercell does not pose a restriction for the FCT situation where all molecules in the monolayer are equivalent. However, for ICT, it only allows to model the situation where every other molecule is charged (and not, say, one out of four). Allowing for different ratios between charged and neutral molecules would require the use of even larger supercells, which is presently intractable using hybrid functionals. The precise fraction of charged molecules is, however, inconsequential for the present study, which deals with the conceptual differences between FCT and ICT. Thus, also the limitation to two molecules per supercell does not affect the conclusions. (See also the low-coverage simulations presented in the Supporting Information).

To understand why the use of hybrid functionals is imperative for the description of ICT, one needs to consider how the energy of a molecule evolves with (fractional) charging. For the ideal, exact functional, the total energy of a system is piecewise linear between integer electron numbers (see black lines in Figure $3 a) .^{5,8,27,52-55}$ Since all available exchangecorrelation functionals are approximations, errors arise, which cause deviations from this straight-line behavior. The so-called deviation from straight-line error DSLE ${ }^{11}$ (often referred to as many-electron self-interaction error ${ }^{26,27}$ ) leads to a systematic over- or underestimation 
of the total energy for fractional electron numbers (depending on the functional), even if it does not necessarily affect the energy at integer electron numbers. ${ }^{8,26,27,56-58}$ In semi-local functionals such as PBE, the DSLE consistently leads to an underestimation of the energy for fractionally charged moieties, $8,11,27,56$ and, therefore, to a concave shape in the dependence of the total energy on the particle number (see blue line in Figure 3a).

To understand how this error affects the charge transfer mechanism, one needs to consider the energetics of molecular orbitals. In Kohn-Sham DFT, the energy of an orbital is given by the derivative of the total energy with respect to the filling of the orbital. ${ }^{59}$

$$
\varepsilon=\frac{d E}{d N}
$$

This means that for a DSLE-afflicted functional, the orbital energy will generally depend on its occupation. For the case of a semi-local functional with a concave curvature of the total energy with respect to the occupation (blue line in Figure 3a), the orbital energy will, therefore, increase (i.e., typically become less negative) as it gets filled (see Figure 3b). Hartree-Fock exhibits the contrary behavior. The total energy of a system with fractional electron numbers is systematically overestimated, ${ }^{35}$ which is again due to the DSLE. In this context it is worth pointing out that, while Hartree-Fock is free from single-electron selfinteraction, the many-electron self-interaction error is still present. ${ }^{27}$ The total energy is curved in a convex form (see red line in Figure 3a) and, therefore, the orbital energy decreases (i.e., typically becomes more negative) with increasing occupation (see Figure 3b). 


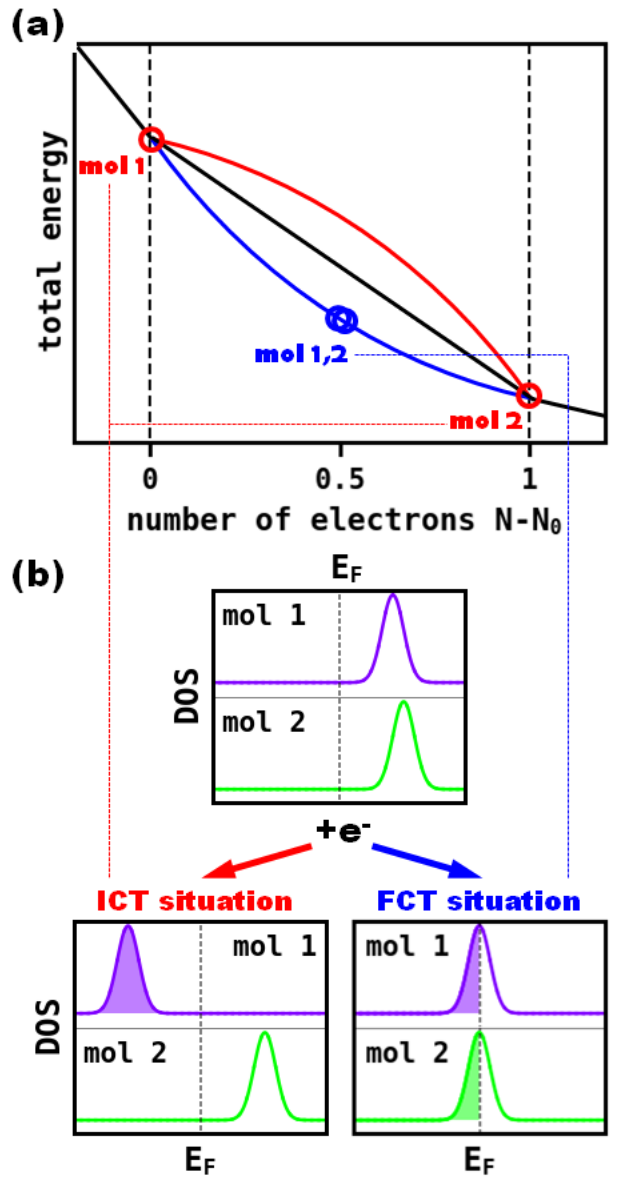

Figure 3: Schematic presentation of over-delocalization and over-localization due to the DSLE. (a) Evolution of the total energy of a system depending on the (fractional) number of electrons it contains. $\mathrm{N}_{0}$ corresponds to the charge-neutral situation. The black line shows the ideal, DSLE free situation where the dependence of the energy on the number of electrons is piecewise linear between integer electron numbers. The blue curve depicts the situation of $\alpha=0$, where overdelocalization of charge appears and the energy at fractional electron numbers is underestimated. The red curve depicts the opposite situation of $\alpha=1$, where the energy for fractional electron numbers is overestimated and strong over-localization of charge occurs. The lowest energy configuration for the two molecule system shown in (b) is marked with red ( $\alpha=1$, ICT) and blue ( $\alpha=0$, FCT) circles. (b) One electron is added to the frontier orbitals of a system containing two molecules. Over-localization shifts the orbitals apart and leads to one full and one empty orbital upon charging, which results in the ICT case (red). Over-delocalization, on the other hand, results in two half filled orbitals and therefore an FCT situation (blue). 
Knowing this dependence we can argue what will happen in a charge transfer system with two molecules. Even when the molecules are notionally identical, their LUMOs will not be perfectly aligned, e.g., as a result of dynamic disorder. As soon as a minute amount of charge is transferred to one of the molecules, its lowest-lying unoccupied orbital will become partly occupied. In case of a semi-local functional $(\alpha=0)$ this results in an upwards shift of the orbital energy until it comes into resonance with the lowest-lying empty orbital of the other molecule. This charge-transfer-induced destabilization of the respective orbital inevitably results in an even distribution of charge between both molecules. This resembles the FCT situation (see Figure 3b). Note, however, that the FCT charge distribution does not originate from the physical situation, as no hybridization (either between the molecules or to a substrate) occurs. Rather, it is purely a numerical artifact, which arises from the dependence of the orbital energy on its occupation.

For this case, the energetically ideal configuration for the transfer of one electron is marked by the blue circles in Figure 3a. As a consequence, strongly hybridizing systems, which are expected to exhibit FCT are qualitatively correctly described in the framework of semi-local functionals. The tendency to over-delocalize charges, ${ }^{26,27,29}$ however, prevents a successful simulation of ICT systems. ${ }^{20}$

Conversely, for Hartree-Fock (or hybrid functionals comprising a large amount of HartreeFock exchange) adding charge to an unoccupied orbital of an adsorbed molecule will result in a downshift of the orbital in energy. The orbital is, therefore, stabilized compared to the corresponding orbitals of neighboring molecules (see Figure $3 b$ ). Thus, the orbital of one molecule must become fully charged before charge can be added to orbitals of another molecule. Consequently, for the model case of one electron being transferred per two molecules, the total energy is minimized by fully charging one molecule while leaving the 
other one neutral. This is depicted by the red circles in Figure 3a. Therefore, in Hartree-Fock and for large $\alpha$, the DSLE tends to over-localize charges favoring the ICT scenario.

Due to the contrasting behavior of the aforementioned methods, it is possible to construct hybrid functionals that aim at compensating the DSLE. This can be done by complementing semi-local with Hartree-Fock exchange (see Equation 1), ${ }^{35}$ where the residual DSLE depends on the applied mixing ratio expressed by the parameter $\alpha$. We note that also other methods exist to compensate for the DLSE, including (empirical) self-interaction correction schemes or range-separated hybrid functionals with either a general parameterization, such as the popular CAM-B3LYP 60,61 or the HSE ${ }^{62,63}$ functional, or optimally tuned functionals with a system-specific parameterization. ${ }^{58,64-69}$ However, even if some these approaches might be computationally cheaper, they contain more parameters than a simple, global hybrid functional, which makes them harder to interpret physically. Therefore, they are not in the scope of the present work.

The above discussed cases emphasize the relevance of the parameter $\alpha$, which determines how the energies of the frontier orbitals depend on (partial) occupation. ${ }^{70}$ Irrespective of the actual physical properties of an interface, low values of $\alpha$ (or no Hartree-Fock exchange mixed in at all) will promote an FCT situation, while sufficiently high amounts of $\alpha$ will favor an ICT situation in the simulations. Under certain circumstances, the value of $\alpha$ can, therefore, be the deciding factor whether an FCT or an ICT situation is found in a DFT calculation.

As the DSLE-free situation marks the transition point between over-localization and overdelocalization, it is worthwhile identifying the corresponding value of $\alpha$ (denoted here as $\left.\alpha^{*}\right) \cdot{ }^{11,71}$ Thus, in the following we will identify this transition point for different interfaces and will also discuss how its value is influenced by the physical situation. Moreover, we will 
describe which information can be gained by varying $\alpha$ and under which circumstances the choice of $\alpha$ has little or no qualitative impact on the obtained results.

\section{Results and Discussion}

\subsection{Impact of $\alpha$ on the Electronic Structure of TFBQ in the Gas Phase}

The first system for which we determine $\alpha^{*}$ is the TFBQ molecule in the gas phase. Here, we discuss two possible approaches, namely enforcing the straight-line condition and comparing the LUMO energies to the electron affinity of the molecule.

Straight-line condition. To assess the straight-line condition, we calculate the LUMO energies of the molecule in gas phase as a function of $\alpha$. We consider the neutral molecule as well as the molecule charged by half an electron and by one electron. In the latter case the considered orbital corresponds to the SOMO of the TFBQ anion. The corresponding values are shown in Figure 4a, where we interpolate linearly between the data points, which is consistent with the results for gas phase molecules in previous works. ${ }^{11,70}$ It follows from Equation 2 that the straight-line condition is met when energy of an orbital becomes independent of its occupation. For low values of $\alpha(0.00,0.25$ and 0.50$)$ we find an increase of the orbital energy with filling, while for higher values of $\alpha(0.75,1.00)$ the orbital energy decreases. The slope $d \varepsilon / d N$ is determined from a linear fit and is plotted as a function of $\alpha$ in Figure $4 \mathrm{~b}$. By interpolation, we deduce the value of $\alpha^{*}=0.62$ to fulfill the straight-line condition. This value might appear somewhat high compared to the amount of Hartree-Fock exchange employed in many commonly used functionals. In this context, it is useful to remember that there is a relationship between the fraction of Hartree-Fock exchange and the Hubbard $U$ term for the on-site charging energy (see Introduction). ${ }^{31-33}$ Physically, this 
parameter describes that it is more difficult to ionize a molecule a second time (even if the second charge is put into the same orbital), due to Coulomb repulsion between the two charges. This repulsion is larger for more confined orbitals, i.e. small molecules typically require large values of $U$ (and, thus, $\alpha$ ). The value of $\alpha^{*}=0.62$ is also very well in line with the values of $\alpha^{*}$ around 0.7 obtained for other small molecules like $\operatorname{TCNE}^{20}, \mathrm{TCNQ}^{11}$ and benzene. ${ }^{71}$ 
(a)

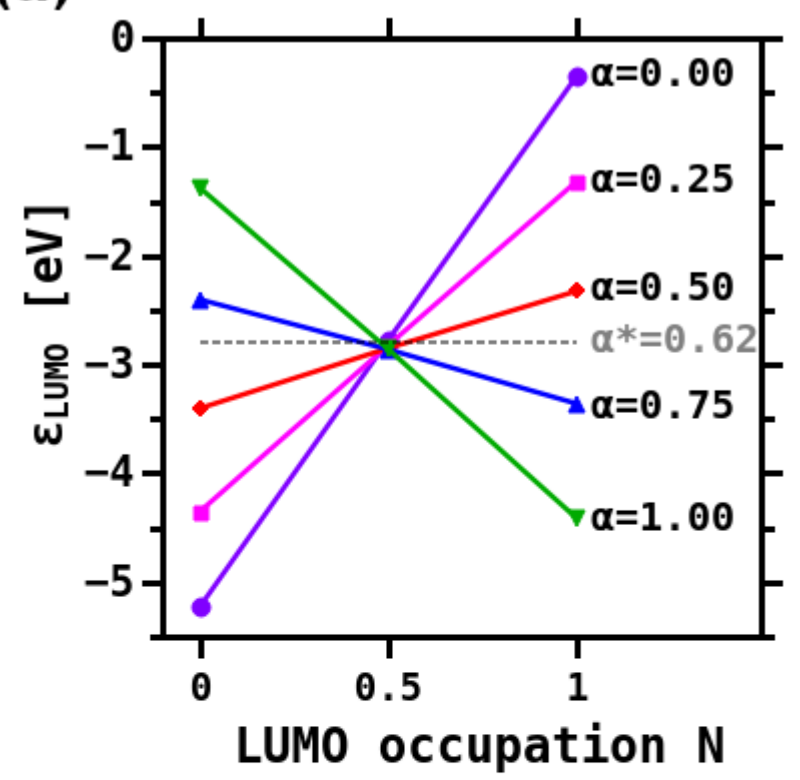

(b)

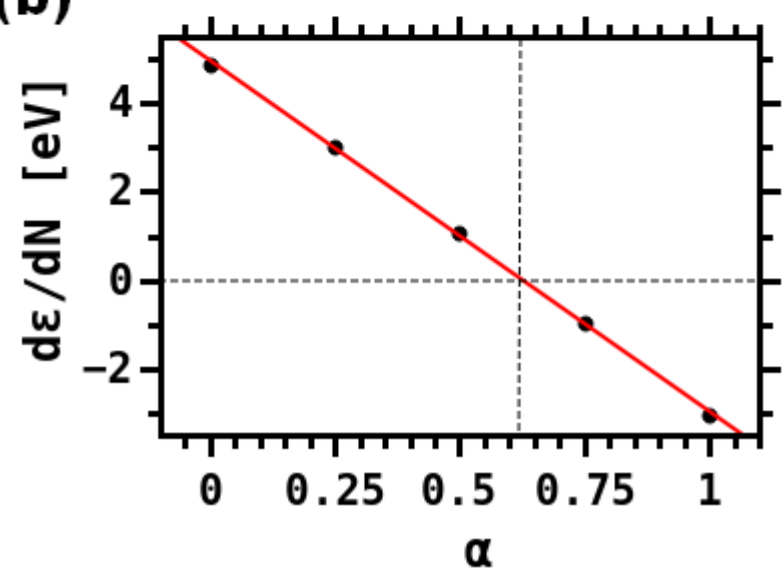

Figure 4: (a) Dependence of the LUMO energy $\varepsilon$ on its occupation in the gas phase TFBQ molecule as a function of the amount of Hartree-Fock exchange $\alpha$. Three charging levels $(\mathrm{N}=0, \mathrm{~N}=0.5$ and $\mathrm{N}=1)$ have been considered and the value $\alpha^{*}$ is marked by the grey, dashed line. (b) Dependence of d $d / d N$ (the change of the LUMO energy with charging) as a function of $\alpha$. By applying a linear fit, a value $\alpha^{*}=0.62$ for $d \varepsilon / d N=0$ (i.e., the straight-line condition) is obtained $\left(R^{2}=0.9994\right)$.

Comparison of electron affinity and LUMO energy. An alternative approach for obtaining a DSLE-free functional is to tune $\alpha$ such that the (negative) energy of the highest occupied molecular orbital (HOMO) corresponds to the ionization potential of the system. ${ }^{11,72,73}$ As the 
vertical ionization potential of the negatively charged molecular anion is equal to the vertical electron affinity (EA) of the neutral molecule, either of the quantities can be considered. Likewise, in the DSLE-free case, the energy of the SOMO of the anion equals the energy of the LUMO' of the neutral molecule. ${ }^{11}$ Consequently, a viable tuning procedure would also be to match the electron affinity of the neutral molecule with its LUMO energy. As this line of arguments is independent of the underlying molecular geometries, it can also be applied to adiabatic quantities (i.e., relaxing geometries of the charged states, as will be done in the following). Such tuning procedures have been applied frequently and have yielded an improved prediction of several electronic properties. ${ }^{68,71,73,77}$

To obtain the electron affinity, we calculate the energy differences between the neutral molecule and the singly negatively charged system.

$$
E A=E_{\text {neutral }}-E_{\text {anion }}=-\varepsilon_{\text {LUMO }}
$$

This procedure is denoted as the $\triangle \mathrm{SCF}$ approach. ${ }^{70,78-80}$ For all applied hybrid functionals considered here, EA is found to vary by less than $0.1 \mathrm{eV}$, which is in line with previous findings that the $\triangle \mathrm{SCF}$ electron affinity does not strongly depend on the functional. ${ }^{70}$ The average electron affinity amounts to $2.84 \mathrm{eV}$. The TFBQ LUMO energies as a function of $\alpha$ are shown in Figure 5. From this plot it can be concluded that for matching the LUMO energy with the average electron affinity (dashed line), a value of $\alpha^{*}=0.63$ is required. This is consistent with the value $\alpha^{*}=0.62$ found above enforcing the straight-line condition.

\footnotetext{
IIn the exact functional, the LUMO changes its energy upon infinitesimal filling due to the so-called derivative discontinuity. ${ }^{53,74-76}$ Since the semi-local and hybrid functionals do not exhibit this behavior, we neglect this detail here.
} 


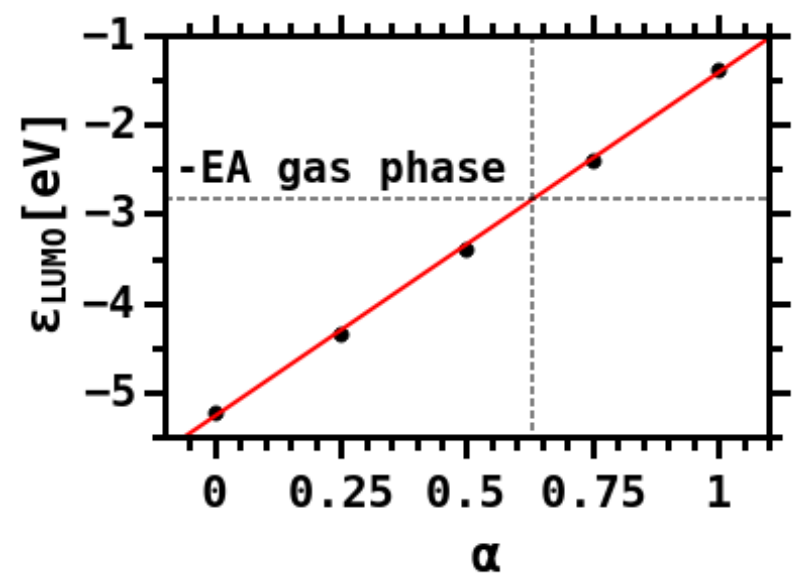

Figure 5: LUMO energies of the TFBQ molecule calculated with different values of $\alpha$. The dashed lines mark the negative electron affinities EA and the corresponding values of $\alpha^{*}$ calculated for the gas phase (linear fit of LUMO energies: $R^{2}=0.9990$ )

\subsection{Impact of $\alpha$ on the Electronic Structure of the TFBQ/ $\mathrm{Cu}_{2} \mathrm{O}$ Interface}

Knowing $\alpha^{*}$ for the TFBQ molecule in the gas phase, the question arises how this value compares to $\alpha^{*}$ for an interface with a monolayer of TFBQ molecules adsorbed on a surface. We first consider adsorption on $\mathrm{Cu}_{2} \mathrm{O}$, where we expect only little hybridization and, therefore, anticipate an ICT situation. Here, the modified $\alpha^{*}$ is estimated based on the straight-line condition for the lowest unoccupied molecular state at the interface (which, for the sake of consistency, we continue to call LUMO in the following). This is analogous to the first strategy employed for the gas phase molecule. As a complementary approach, we compare the energies for the ICT and FCT scenarios for different values of $\alpha$ and from that determine $\alpha^{*}$.

Prior to that, it is useful to discuss how $\alpha^{*}$ should change due to the substrate. For the following semi-quantitative argument, we neglect the impact of hybridization and different geometric relaxations at the interface compared to the gas phase, but rather focus on the 
role played by the dielectric screening of the additional negative charge through the substrate. The screening causes an overall increase of the electron affinity. Conceptually, this should also be reflected in the positions of the unoccupied states derived from the LUMO of the neutral adsorbate. It has, however, been shown that neither semi-local nor hybrid functionals capture this effect. ${ }^{81-84}$ Therefore, in a situation with no hybridization and no geometric distortions upon adsorption, the LUMO energies of uncharged molecules are not affected by the presence of a surface. Consequently, a first approximate value for $\alpha^{*}$ at the interface can be obtained by comparing the electron affinity of the interface with the LUMO energy of the gas phase molecule.

Ideally, we would like to calculate the electron affinity on the surface via the $\triangle \mathrm{SCF}$ approach, i.e. as energy difference of the charged and the uncharged moiety. This is, however, not directly possible with periodic boundary conditions, since charging of the unit cell would lead to a diverging energy. In practice, this can be avoided by assuming a homogenous charged background in the unit cell. Especially for interfaces, such an approach does not solve the problem since then the charging energy would diverge with increasing the width of the vacuum gap. In principle, the GW method (which provides charged excitations) would be a viable alternative approach. However, the results of the typically conducted non-selfconsistent GW corrections depend on the starting point, i.e., the underlying DFT calculations and therefore the functional. ${ }^{85,86}$ Moreover, for the interfaces investigated in this work, GW is presently far too costly. It has, however, been discussed in the literature that screening in comparable interface systems increases the electron affinity by approximately $1 \mathrm{eV} .^{81} \mathrm{By}$ comparing this value to the LUMO energies derived in gas phase (see Figure 5) we conclude that surface screening will significantly lower the required value of $\alpha^{*}$. These considerations 
show that the presence of a screening substrate can be expected to significantly reduce the amount of Hartree-Fock exchange necessary to realize a DSLE-free situation. Considering that the screening energy scales with the dielectric substrate, even more significant reductions of $\alpha^{*}$ can be expected for molecules adsorbed on highly polarizable substrates.

Straight-line condition. To obtain a quantitative estimate for $\alpha^{*}$ it is necessary to calculate the actual interface, where not only screening effects, but also geometric rearrangements and a possible hybridization between TFBQ and $\mathrm{Cu}_{2} \mathrm{O}$ states influences in the LUMO energies. Then, a value of $\alpha^{*}$ including all adsorption-induced contributions can be derived from enforcing the straight-line condition. In analogy to section 4.1., this corresponds to finding the value of $\alpha$ for which the LUMO (respectively, SOMO) energy becomes independent of the filling of the state.

To be able to directly compare LUMO and SOMO energies, the ideal strategy is to calculate both a charged and a neutral molecule in one unit cell. To achieve that, we choose a supercell containing two TFBQ molecules on a $8 \times 4 \mathrm{Cu}_{2} \mathrm{O}(111)$ substrate as shown in Figure 1 . Note that no calculations applying $\alpha=0$ could be included here, for the reasons discussed in the Methodology section.

When adding an electron to the unit cell to fill one of the LUMOs, the charge has to be compensated by a positive charge without breaking the translational symmetry within the unit cell. Thus, a positively charged plate is positioned below the $\mathrm{Cu}_{2} \mathrm{O}$ substrate (details see Supporting Information). ${ }^{87,88}$ This results in a situation which is physically equivalent to $\mathrm{n}$ doped $\mathrm{Cu}_{2} \mathrm{O}$, where free charges are available in the conduction band and can be transferred to the molecules. The additional electron can be donated to either one or both of the electron-accepting TFBQ molecules. For all hybrid functionals applied here it is possible to 
achieve a situation, where the electron is localized on one molecule while the second molecule remains charge neutral. The energies of the (essentially non-dispersing band derived from) the LUMO of the neutral molecule and of (the band derived from) the SOMO of the charged molecule are shown in Figure 6a. A value of $\alpha=1.00$, as applied for the gas phase could not be considered for the surface, since in this case we were unable to converge the $\mathrm{Cu}_{2} \mathrm{O}$ substrate. In contrast to the gas phase situation, where only high values of $\alpha$ lead to a decrease in LUMO energy with charging, on the surface we find a decrease for all tested functionals. By extrapolating $d \varepsilon / d N$ as a function of $\alpha$ (Figure $6 b$ ), the value fulfilling the straight-line condition can be determined to $\alpha^{*}=0.10$. Consistent with the above considerations regarding screening effects, this value is significantly lower than in the gas phase. Consequently, on the surface a calculation with $a=0.10$ or higher will favor an ICT situation, while for lower values the FCT scenarios if favored. Therefore, the application of the gas phase value of $\alpha^{*}(0.63)$ to the surface situation will create a significant risk of overlocalizing charges and strongly favor ICT. 


\section{(a)}
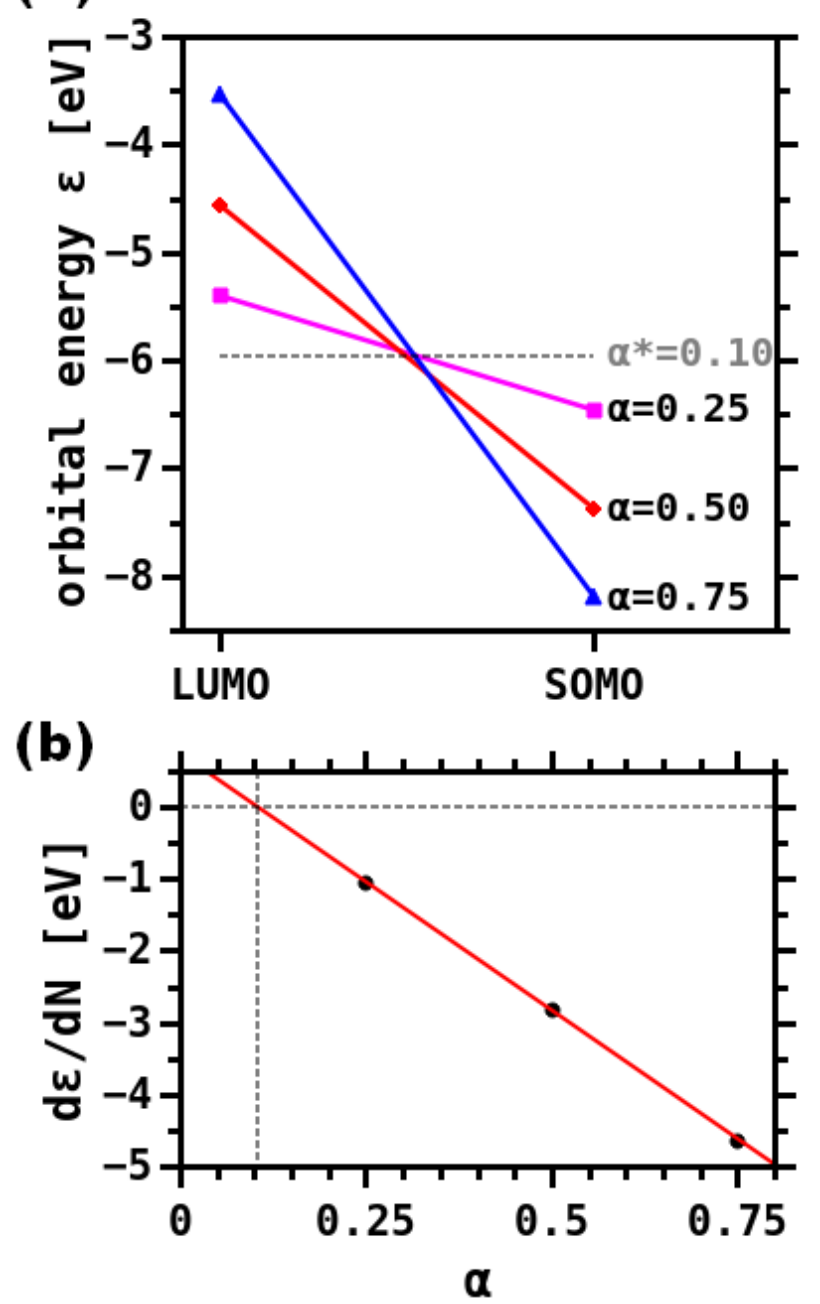

Figure 6: Orbital energies of two TFBQ molecules adsorbed on the $\mathrm{Cu}_{2} \mathrm{O}(111)$ surface in an $8 \times 4$ surface unit cell. (a) The presence of one charged and one neutral molecule in the cell allow the direct comparison of LUMO and SOMO energies for three different values of $\alpha$. The energies are given relative to the upper vacuum level. (b) Change of LUMO energy for all considered values of $\alpha$. By applying a linear fit the value of $\alpha^{*}=0.10$ for $d \varepsilon / d N=0$ is derived $\left(R^{2}=0.9998\right)$.

Notably, $\alpha^{*}=0.10$ is also significantly below the frequently applied value $\alpha=0.25$ (as used e.g. in PBEO $3,30,35)$, suggesting that "standard" hybrid calculations would also be biased towards charge over-localization and, thus, towards ICT in the present case. As we intent to investigate the influence of the surface adsorption, we want to exclude that 
the derived $\alpha^{*}$ is influenced strongly affected by the presence of neighboring (screening) molecules. Therefore, we further reduced the molecular coverage to $1 / 4$ of the previous value. As described in the Supporting Informtion, this yields $\alpha^{*}=0.08$, showing that the considered coverage with one molecule per $126 \AA^{2}$ is low enough to render the value of $\alpha^{*}$ determined above insensitive to the presence of neighboring molecules.

Charge transfer mechanism for different values of $\alpha$. The unit cell containing two molecules conceptually allows the simulation of the ICT-induced coexistence of two differently charged molecules on the surface. This enables a direct investigation of the charge transfer mechanism.

As a first step, we consider the DOS projected onto the molecules, which is calculated with different values of $\alpha$ and is shown in Figure 7. For $\alpha=0.00$, the charge is evenly distributed between both molecules and neither symmetry breaking nor spin splitting appears. Thus, this situation can be described by the FCT model. Conversely, for $\alpha=0.50$ and $\alpha=0.75$ the projected DOS exhibits symmetry breaking between molecule 1 and molecule 2 and for the charged molecule the DOS becomes clearly spin-polarized. These calculations can be assigned to the ICT scenario. We note that early discussions of the ICT scenario invoked polaron levels in the gap (sometimes also denoted as ICT-levels) as relevant for the charge transfer. Our calculations clearly show that there is no in-gap state. Rather, as found also by other authors, ${ }^{51,89,90}$ the LUMO of the charged molecule splits into a SOMO and a SUMO state, where the SUMO is found at higher energies than the LUMO of the neutral molecule. For $\alpha=0.25$, the situation is less clear. We obtained two solutions, where the projected DOSes shown in Figure 7 imply that one corresponds to an FCT and the other one to an ICT solution. Notably, these differing results are obtained by different initializations of the calculations: 
While the default initialization (which in FHI-aims is a superposition of the spherical atomic charge densities) ${ }^{36}$ leads to the FCT scenario, any asymmetric magnetic initialization of molecules 1 and 2 converges to the ICT solution. 


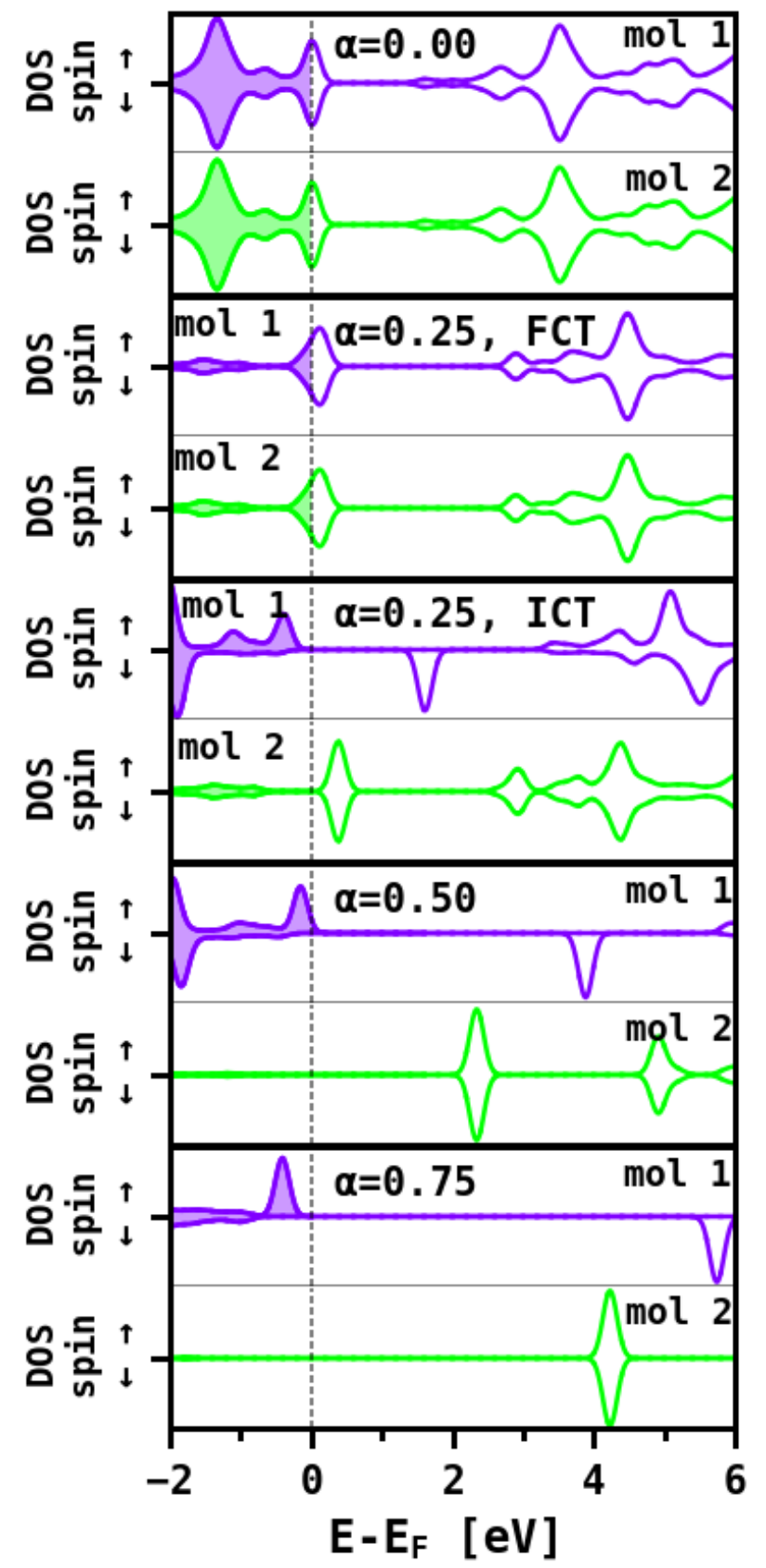

Figure 7: DOSs projected on molecules 1 (purple) and 2 (green) for all applied functionals. For $\alpha=0.25$ both FCT and ICT solution are shown. Spin-up and spin-down DOSs are shown in positive and negative direction in arbitrary units. The energy axis is aligned to the Fermi energy (and in case of no DOS at the Fermi level, to the valence band maximum of the underlying $\mathrm{Cu}_{2} \mathrm{O}$ substrate).

To further analyze these observations, we establish charge localization on the molecules as an observable to distinguish between the charge transfer mechanisms. This is done by 
calculating the charges of individual molecules and defining a charge localization parameter, L, as:

$$
L=\frac{Q_{m o l 1}-Q_{m o l 2}}{Q_{m o l 1}+Q_{m o l 2}}
$$

When the charge is evenly distributed between both molecules, the localization parameter becomes zero, which corresponds to an ideal FCT case. Charging of one molecule with the other one remaining neutral results in a localization of $L=1$ (or $L=-1$ ). This corresponds to an ideal ICT situation.

However, determining the charges faces two challenges: First of all, it is important to keep in mind that atomic or molecular charges are not unambiguously defined observables, and different partitioning schemes usually lead to varying results. Additionally, although TFBQ interacts only weakly with the $\mathrm{Cu}_{2} \mathrm{O}$ substrate, there is a small, but non-zero hybridization between the substrate and deeper-lying $\sigma$ orbitals. As a result of these two complications, an "uncharged" molecule (with no charge transfer into the LUMO) may become nominally positively charged. In the present case, the high- $\alpha$ ICT solutions exhibit such positive charging if the LUMO of the molecule remains empty. This leads to the non-intuitive situation of a nominal charge localization $L>1$. For the determination of the localization parameter, here we use Mulliken charges $^{91}$ on the molecules. In order to exclude positive charging due to hybridization and/or Mulliken artifacts, we set the positively charged molecule to $Q=0$. In the Supporting Information, we report the results for three alternative strategies: Considering only the LUMO occupation of the two molecules, considering the full Mulliken charges, or partitioning via Hirshfeld ${ }^{92}$ charges. We emphasize that for all these choices, the conclusions drawn hereafter prevail. 
Figure 8 shows the absolute value of $L$ for TFBQ on $\mathrm{Cu}_{2} \mathrm{O}$ for different values of $\alpha$. For $\alpha=0.00$, $L=0$ consistent with the FCT situation discussed above. For $\alpha=0.50$ and $\alpha=0.75$ a value of $L=1$ is obtained with one molecule being charged and the other one remaining neutral. For $\alpha=0.25$, the FCT solution exhibits $L=0$, while for the second solution $L=0.8$ is obtained. The meaning of $L$ being neither 1 nor 0 as well as the reason for the coexistence of FCT and ICT solutions for $\alpha=0.25$ will become clear from the following discussion of the relative energetics of the FCT and ICT scenarios.

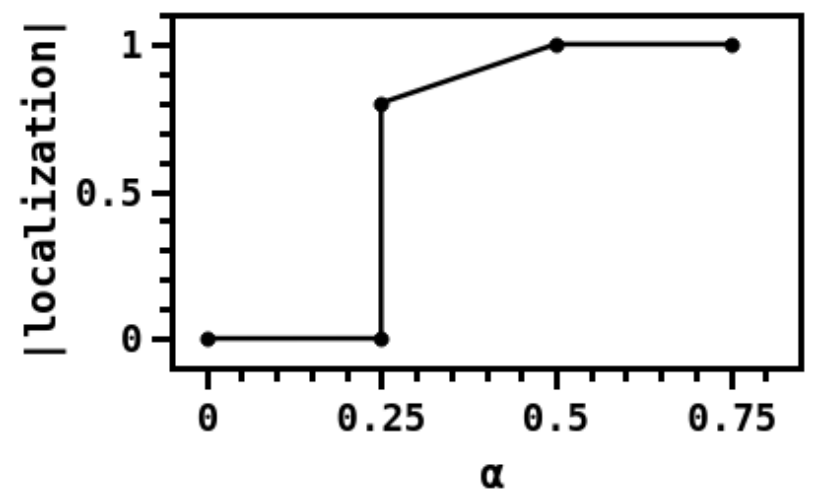

Figure 8: The absolute value of localization of charge for two TFBQ molecules on $\mathrm{Cu}_{2} \mathrm{O}$ for different amounts of $\alpha$. For $\alpha=0.25$, both obtained solutions are shown.

Relative energies of FCT and ICT solutions. When we reconsider the energy progression in Figure $3 a$, we see that the relative energies of the FCT and ICT solutions have to change upon varying $\alpha$. For our system (two TFBQ molecules on $\mathrm{Cu}_{2} \mathrm{O}+$ one extra electron per unit cell), this leads to relative energies as schematically shown in Figure 9.

There are two degenerate ICT solutions corresponding to the additional charge localized either on the first $(L=+1)$ or on the second molecule in the unit cell $(L=-1)$. They are denoted as ICT 1 and ICT 2 in Figure 9. 
The ideal FCT solution with both molecules equivalently charged marks the transition point between the ICT solutions and must be at a symmetric position relative to both. For the high$\alpha$ case (red curve in Figure 9), the FCT solution is energetically unfavorable compared to the ICT solutions. However, due to the symmetry of the energy evolution with respect to changing the sign of L, it must be a stationary point on the electronic potential energy surface. Thus, conceptually, it has to be accessible as a stable solution in DFT simulations. In the low- $\alpha$ case, the FCT scenario is the energetic minimum and the ICT solutions do not represent stable, stationary points. It is, therefore, usually not possible to enforce an ICT solution for $\alpha<\alpha^{*}$. This is in line with our results where we are not able to converge an ICT solution for $\alpha=0.00$ even with asymmetric spin initialization.

For $\alpha=0.25$, which is slightly above $\alpha^{*}$, FCT and ICT solutions can be obtained depending on the initialization. Typically, when the symmetry between the molecules is initially broken, the system will converge toward the ICT solution for $\alpha>\alpha^{*}$, as it is implied by the energy progression in Figure 9. Otherwise, the FCT solution will be obtained. Consistent with the above arguments we find that for $\alpha=0.25$ the ICT result is about $0.2 \mathrm{eV}$ per molecule lower in energy.

With increasing values of $\alpha$, convergence of the FCT solution becomes harder and harder, as the energy difference between FCT and ICT solution becomes larger. Minimal distortions from the perfect symmetry of molecules 1 and 2 already drive the calculations toward the much more stable ICT case. This explains why we have not been able to obtain an FCT solution for $\alpha=0.50$ and $\alpha=0.75$. 


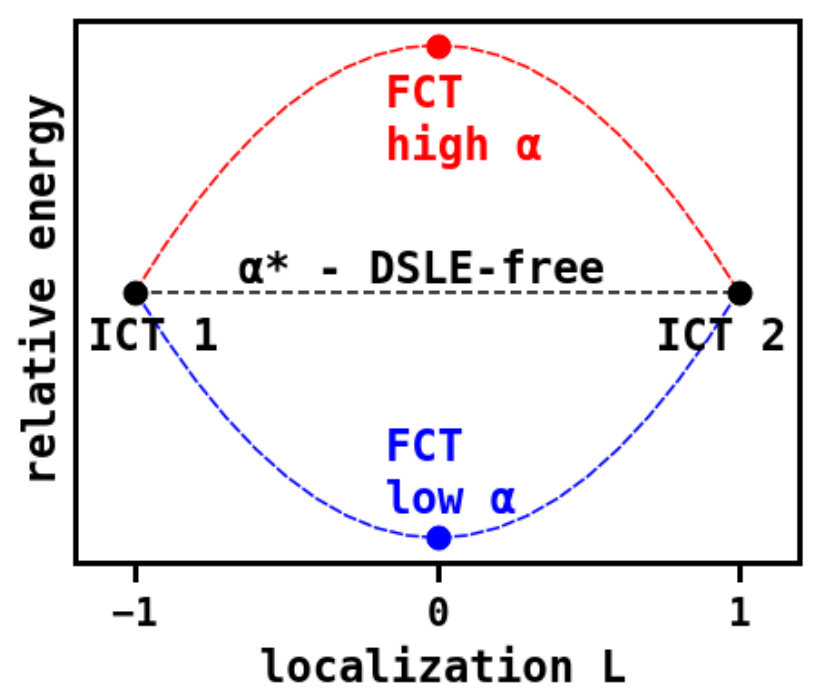

Figure 9: Schematic representation of the relative energies of different solutions, denoted by the associated charge-localization parameter $L$ in the two molecule system. The two ICT solutions, representing charging of the first molecule while the second one remains uncharged and vice versa, are degenerated in energy. For high values of $\alpha$, the FCT solution is higher in energy than the ICT solutions (red), while for low values of $\alpha$, it is lower (blue). At the transition value $\alpha^{*}$, which marks the DSLE-free situation, FCT and ICT solutions are at the same energy. Note that the dashed lines depicting the evolution of the energy with $L$ are idealized. For example, the FCT solution at high values of $\alpha$ is not necessarily a maximum, but might also correspond to a local minimum.

Figure 9 also gives insight into the situation where an amount of Hartree-Fock exchange corresponding to $\alpha^{*}$ is used, i.e., in the case of a DSLE-free calculation. Then, the energies of FCT and ICT solutions must be degenerate. Therefore, also every linear combination of these solutions is a valid solution with the same energy. This leads to two problems: First, charge can be shifted within the monolayer at no energetic cost, making the SCF notoriously difficult to converge (i.e., while the energy will be converged immediately, the change of the density will not). Secondly, even when convergence can be achieved, the charge-distribution is basically arbitrary, and cannot be assigned to either FCT or ICT. This is in line with the above results for $\alpha=0.25$, which is comparably close to the straight-line value $\alpha^{*}$. We find an ICT 
solution where the localization amounts to only 0.8 instead of 1 . As the FCT and ICT solutions are already quite close in energy at this point, we find a solution which is not the ideal ICT scenario, but slightly deviates from it.

Estimation of $\alpha^{*}$ by comparing energies of the FCT and ICT solutions. The fact that at $\alpha^{*}$ the FCT and ICT solutions are degenerate provides an additional strategy for estimating the amount of Hartree-Fock exchange required for a DSLE-free functional. As discussed above, it should be conceptually possible to simulate FCT scenarios for all values $\alpha>\alpha^{*}$. As minimal symmetry distortions prevent the DFT calculations from readily converging into the FCT solution at high values of $\alpha$, in such cases symmetry must be enforced. This can be achieved by performing the simulations in a non-spin-polarized manner.

The resulting energy differences between FCT and ICT solutions for different values of $\alpha$ are shown in Figure 10. From these we can estimate a value of $\alpha^{*}=0.03$, where both solutions exhibit equivalent energies. This is satisfactorily in line with our previous results of $\alpha^{*}=0.10$ as obtained from enforcing the straight-line condition and again well below the commonly applied $\alpha=0.25$. 


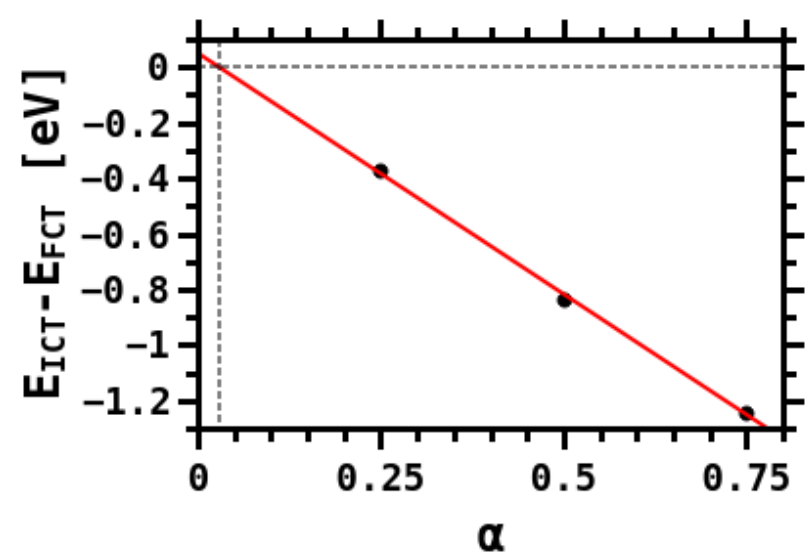

Figure 10: Energy difference between the ICT and FCT solutions as a function of $\alpha$. The transition point where both solutions exhibit the same energy which deduced from the linear fit depicted in red lies at $\alpha^{*}=0.03\left(R^{2}=0.9986\right)$

\subsection{Impact of $\alpha$ on the Electronic Structure of the TFBQ/Cu Interface}

Before discussing how $\alpha$ should be chosen for a given system, we look at a physically different situation, namely the adsorption of TFBQ on $\mathrm{Cu}(111)$. Above we found that for the TFBO/Cu $2 \mathrm{O}$ interface, the ICT scenario can always be simulated by applying a sufficiently high value of $\alpha$. This raises the question, whether this effect is system-independent and whether an ICT solution can be found for every system as long as $\alpha$ is high enough. Considering that $\alpha^{*}$ ought to be reduced by screening effects (vide supra), on metallic surfaces a value of $\alpha^{*}$ even smaller than on $\mathrm{Cu}_{2} \mathrm{O}$ should be expected due to the particularly strong screening. Conversely, in a situation in which organic molecules are in direct contact with a metal surface, one would expect an FCT situation due to hybridization effects of the molecules with the substrate. Thus, from the above discussion it is a priori not clear how $\alpha$ affects the calculated charge transfer mechanism on a bare metal substrate.

Therefore, we simulate the adsorption of TFBQ on $\mathrm{Cu}(111)$. To be able to identify ICT behavior, again a unit cell containing at least two TFBQ molecules is required and an $8 \times 4$ surface cell in analogy to the one considered for $\mathrm{Cu}_{2} \mathrm{O}$ is applied. The resulting molecule- 
projected DOSs for different values of $\alpha$ are shown in Figure 11 and the Mulliken charges per molecule are shown in Table 2.

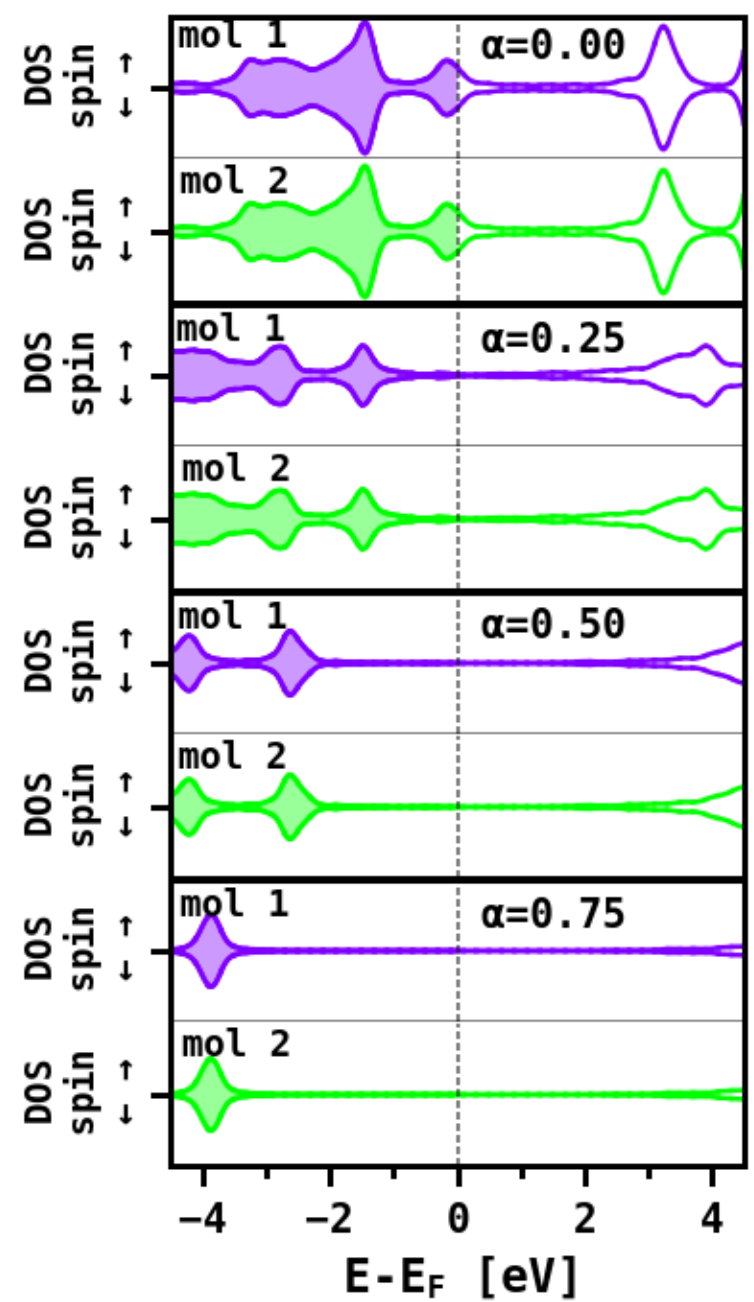

Figure 11: DOSs projected on molecules 1 (purple) and 2 (green) for all applied functionals. Spin-up and spin-down DOSs are shown in positive and negative direction in arbitrary units. The energy axis is aligned to the Fermi energy. 
Table 2: Mulliken Charges in Electrons per Molecule for Two TFBQ Molecules on the Cu(111) Surface for Different Values of $\alpha$.

\begin{tabular}{|l|c|c|c|c|}
\hline & $\alpha=0.00$ & $\alpha=0.25$ & $\alpha=0.5$ & $\alpha=0.75$ \\
\hline $\begin{array}{l}\text { Mulliken charge } \\
\text { molecule 1 [electrons] }\end{array}$ & 0.61 & 0.88 & 1.01 & 1.12 \\
\hline $\begin{array}{l}\text { Mulliken charge } \\
\text { molecule 2 [electrons] }\end{array}$ & 0.61 & 0.88 & 1.01 & 1.12 \\
\hline
\end{tabular}

The PBE simulation shows typical FCT behavior, with both molecules exhibiting equivalent partial charging of the LUMO and, therefore, non-zero DOS at the Fermi level. In addition, both molecules get equally charged with 0.61 electrons. All calculations employing $\alpha>0$ exhibit fully charged LUMOs for both molecules, which lie clearly below the Fermi level and the molecule-projected DOS at the Fermi level drops to zero. With higher amounts of $\alpha$, the LUMOs shift to lower energies and are completely filled (in the spin-up and spin-down channels). This suggests that two electrons per molecule have been transferred. The overall transferred charge, however, amounts to approximately one electron per molecule. This can be explained by back-donation from nominally deeper-lying orbitals that hybridize particularly strongly with the substrate..$^{13,93}$

The net transfer of one electron per molecule and the vanishing projected DOS at the Fermi edge point towards an ICT scenario. This is, however, ruled out by the identical structures and charges of the two molecules and by the identical occupations of the spin channels. Furthermore, the amount of charge on each molecule continuously increases with increasing $\alpha$, which would also not be the case in an ICT scenario.

The fundamental difference between the previously discussed adsorption on $\mathrm{Cu}_{2} \mathrm{O}$ and that on $\mathrm{Cu}$ lies in the hybridization between molecular and substrate states. On $\mathrm{Cu}_{2} \mathrm{O}$, molecule 
and substrate are electronically largely decoupled, which leads to an ICT scenario even at low values of $\alpha$. Conversely, on Cu hybridization between molecule and substrate states appears even at high values of $\alpha$ in spite of the tendency to over-localize charges. Such a strong metalmolecule coupling is in line with the reduced adsorption height the molecule exhibits on $\mathrm{Cu}$ in comparison to $\mathrm{Cu}_{2} \mathrm{O}$. (The maximum height difference is found for $\alpha=0.75$, where charged TFBQ adsorbs $0.27 \AA$ lower on $\mathrm{Cu}$ than on $\mathrm{Cu}_{2} \mathrm{O}$ relative the topmost $\mathrm{Cu}$ layer.)

We, therefore, conclude that the strong hybridization appearing between TFBQ and the metallic surface outweighs the effect of stronger screening and causes FCT to appear. Notably, this effect can be described adequately in DFT, with semi-local as well as with hybrid functionals.

\section{Summary and Conclusions: Suggested Choice of $\alpha$}

In this contribution we focus on the interplay between the charge transfer mode across inorganic/organic interfaces and the choice of the DFT functional (i.e. the amount of HartreeFock exchange $\alpha$ ) used to model it. This is discussed for the example of a monolayer of TFBQ molecules adsorbed on semiconducting $\mathrm{Cu}_{2} \mathrm{O}$ and metallic $\mathrm{Cu}$ substrates. We show that hybrid functionals exhibit a transition point $\alpha^{*}$ between spurious over-localization and overdelocalization of charges favoring either fractional charge transfer (FCT, i.e., all molecules being equally charged) or integer charge transfer (ICT, i.e., the coexistence of charged and uncharged molecules on the surfaces).

We describe how $\alpha^{*}$ can be determined in various ways for both the molecule and the interface and show that it is massively reduced by a screening substrate. Our results indicate that the weaker the screening (i.e., the lower the dielectric constant), the higher are the 
expected values of the transition point $\alpha^{*}$. As far as the actually calculated charge transfer mechanism is concerned, the choice of $\alpha$ relative to $\alpha^{*}$ fundamentally changes the situation on the semiconducting substrate. This is in contrast to the adsorption on $\mathrm{Cu}(111)$, where the functional does not qualitatively change the charge transfer mechanism. Still, even there it changes the amount of transferred charge and, therefore, influences associated properties, such as the geometry and the adsorption-induced work function change.

This leaves the question, how to ideally choose $\alpha$ in the case of weak hybridization. For the example of TFBQ on $\mathrm{Cu}_{2} \mathrm{O}(111)$ discussed here, the comparably low value of $\alpha^{*} \approx 0.1$ that we found implies that most commonly applied hybrid functionals will result in an ICT scenario. This outcome is, however, not necessarily predictive.

It is possible to obtain both the FCT and the ICT solution for values $\alpha>\alpha^{*}$, which is due to the symmetry of the wave function. However, in that case the ICT solution is always energetically favorable and, therefore, the relative energies of the solutions are not authoritative.

Then, one might be tempted to conclude that the DLSE-free situation $\alpha=\alpha^{*}$ would be the ideal choice for reliably predicting the charge transfer properties of a given interface. This is, however, not the case. At $\alpha^{*}$, by definition, the LUMO energies of the molecules in the layer are independent of their occupation. Thus, shifting charge from one molecule to another does not cost energy (geometry relaxations notwithstanding). As a result, every possible localization of transferred charge in the layer results in the same total energy. Consequentially, the charge distribution found by the iterative SCF procedure at $\alpha^{*}$ is determined by numerics and basically arbitrary. It follows from the above that at $\alpha^{*}$ ICT and FCT must have the same energy. In hindsight, this is not surprising. There is, in principle, a multitude of ICT solutions, i.e. the "charged overlayer" can hop from one site to another. 
The ensemble average (either time averaged or spatially averaged) of these ICT solutions is equivalent to an FCT charge distribution. This is formally equivalent to the original derivation of the DLSE principle by Perdew et al., ${ }^{53}$ which rationalized fractional electron numbers by statistical mixtures.

Thus, we arrive at the (somewhat disappointing) conclusion that there is no semi-local or hybrid functional that is universally predictive for the charge transfer mechanism at weakly interacting interfaces. Also, there appears to be no universally valid recipe that allows constructing such a functional for a specific material combination.

This means that in order to be able to draw insight from computation, some input from experiment is required to determine the prevalent charge transfer mechanism. The best practice we suggest is to calculate both FCT and ICT for the given interface, e.g., by applying a functional with $\alpha<\alpha^{*}$ and another with $\alpha>\alpha^{*}$, or by enforcing FCT via spin restriction for $\alpha>\alpha^{*}$. By doing so, relevant observables for both scenarios can be calculated. Subsequently, these can be compared to experimentally obtained results. Note that for this comparison timescales may play an important role; i.e. the observables from experiment must be obtained on timescales faster than the supposed charge-hopping times. This is the case, e.g., for photoemission experiments or vibrational spectroscopy.

After the charge transfer mechanism is determined, the corresponding electronic structure can be analyzed to gain valuable insight, e.g., into the origin and strength of bonding, charge transfer, and hybridization. 


\section{Acknowledgements}

Financial support by the Austrian Science Fund (FWF): P28051-N36 and P27631-N36 is gratefully acknowledged. We thank Simon Erker for valuable scientific discussion and computational advice. The computational studies presented have been achieved using the Vienna Scientific Cluster (VSC3).

\section{Supporting Information}

Details on the impact of geometry optimizations, the performed convergence tests, further methodological aspects (spin initialization of molecules and adding charge to the unit cell), calculations with reduced coverage and localization and charge partitioning schemes are provided. This material is available free of charge at http://pubs.acs.org. 


\section{References}

(1) Jensen, F. Introduction to Computational Chemistry, 3rd ed.; John Wiley \& Sons: Chichester, UK, 2017.

(2) Perdew, J. P.; Chevary, J. A.; Vosko, S. H.; Jackson, K. A.; Pederson, M. R.; Singh, D. J.; Fiolhais, C. Atoms, Molecules, Solids, and Surfaces: Applications of the Generalized Gradient Approximation for Exchange and Correlation. Phys. Rev. B 1992, 46 (11), 6671-6687.

(3) Ernzerhof, M.; Scuseria, G. E. Assessment of the Perdew-Burke-Ernzerhof ExchangeCorrelation Functional. J. Chem. Phys. 1999, 110 (11), 5029-5036.

(4) Staroverov, V. N.; Scuseria, G. E.; Tao, J.; Perdew, J. P. Comparative Assessment of a New Nonempirical Density Functional: Molecules and Hydrogen-Bonded Complexes. J. Chem. Phys. 2003, 119 (23), 12129-12137.

(5) Perdew, J. P.; Zunger, A. Self-Interaction Correction to Density-Functional Approximations for Many-Electron Systems. Phys. Rev. B 1981, 23 (10), 5048-5079.

(6) Toher, C.; Filippetti, A.; Sanvito, S.; Burke, K. Self-Interaction Errors in Density-Functional Calculations of Electronic Transport. Phys. Rev. Lett. 2005, 95 (14), 146402.

(7) Ke, S.-H.; Baranger, H. U.; Yang, W. Role of the Exchange-Correlation Potential in Ab Initio Electron Transport Calculations. J. Chem. Phys. 2007, 126 (20), 201102.

(8) Cohen, A. J.; Mori-Sánchez, P.; Yang, W. Insights into Current Limitations of Density Functional Theory. Science 2008, 321 (5890), 792-794.

(9) Sala Fabio Della; Fabiano Eduardo; Laricchia Savio; D’Agostino Stefania; Piacenza Manuel. The Role of Exact-exchange in the Theoretical Description of Organic-metal Interfaces. Int. J. Quantum Chem. 2010, 110 (12), 2162-2171.

(10) Draxl, C.; Nabok, D.; Hannewald, K. Organic/Inorganic Hybrid Materials: Challenges for Ab Initio Methodology. Acc. Chem. Res. 2014, 47 (11), 3225-3232.

(11) Atalla, V.; Zhang, I. Y.; Hofmann, O. T.; Ren, X.; Rinke, P.; Scheffler, M. Enforcing the Linear Behavior of the Total Energy with Hybrid Functionals: Implications for Charge Transfer, Interaction Energies, and the Random-Phase Approximation. Phys. Rev. B 2016, 94 (3), 035140.

(12) Braun, S.; Salaneck, W. R.; FahIman, M. Energy-Level Alignment at Organic/Metal and Organic/Organic Interfaces. Adv. Mater. 2009, 21 (14-15), 1450-1472.

(13) Romaner, L.; Heimel, G.; Brédas, J.-L.; Gerlach, A.; Schreiber, F.; Johnson, R. L.; Zegenhagen, J.; Duhm, S.; Koch, N.; Zojer, E. Impact of Bidirectional Charge Transfer and Molecular Distortions on the Electronic Structure of a Metal-Organic Interface. Phys. Rev. Lett. 2007, 99 (25), 256801.

(14) Duhm, S.; Gerlach, A.; Salzmann, I.; Bröker, B.; Johnson, R. L.; Schreiber, F.; Koch, N. PTCDA on $\mathrm{Au}(111), \mathrm{Ag}(111)$ and $\mathrm{Cu}(111)$ : Correlation of Interface Charge Transfer to Bonding Distance. Org. Electron. 2008, 9 (1), 111-118.

(15) Kröger, I.; Stadtmüller, B.; Stadler, C.; Ziroff, J.; Kochler, M.; Andreas Stahl; Pollinger, F.; Lee, T.-L.; Zegenhagen, J.; Reinert, F.; et al. Submonolayer Growth of Copper-Phthalocyanine on $\mathrm{Ag}(111)$. New J. Phys. 2010, 12 (8), 083038.

(16) Edlbauer, H.; Zojer, E.; Hofmann, O. T. Postadsorption Work Function Tuning via Hydrogen Pressure Control. J. Phys. Chem. C 2015, 119 (48), 27162-27172.

(17) Bokdam, M.; Çakır, D.; Brocks, G. Fermi Level Pinning by Integer Charge Transfer at ElectrodeOrganic Semiconductor Interfaces. Appl. Phys. Lett. 2011, 98 (11), 113303.

(18) Çakir, D.; Bokdam, M.; de Jong, M. P.; Fahlman, M.; Brocks, G. Modeling Charge Transfer at Organic Donor-Acceptor Semiconductor Interfaces. Appl. Phys. Lett. 2012, 100 (20), 203302.

(19) Amsalem, P.; Niederhausen, J.; Wilke, A.; Heimel, G.; Schlesinger, R.; Winkler, S.; Vollmer, A.; Rabe, J. P.; Koch, N. Role of Charge Transfer, Dipole-Dipole Interactions, and Electrostatics in Fermi-Level Pinning at a Molecular Heterojunction on a Metal Surface. Phys. Rev. B 2013, 87 (3), 035440. 
(20) Hofmann, O. T.; Rinke, P.; Scheffler, M.; Heimel, G. Integer versus Fractional Charge Transfer at Metal(/Insulator)/Organic Interfaces: $\mathrm{Cu}(/ \mathrm{NaCl}) / T C N E$. ACS Nano 2015, 9 (5), 5391-5404.

(21) Hollerer, M.; Lüftner, D.; Hurdax, P.; Ules, T.; Soubatch, S.; Tautz, F. S.; Koller, G.; Puschnig, P.; Sterrer, M.; Ramsey, M. G. Charge Transfer and Orbital Level Alignment at Inorganic/Organic Interfaces: The Role of Dielectric Interlayers. ACS Nano 2017, 11 (6), 6252-6260.

(22) Gruenewald, M.; Schirra, L. K.; Winget, P.; Kozlik, M.; Ndione, P. F.; Sigdel, A. K.; Berry, J. J.; Forker, R.; Brédas, J.-L.; Fritz, T.; et al. Integer Charge Transfer and Hybridization at an Organic Semiconductor/Conductive Oxide Interface http://pubs.acs.org/doi/full/10.1021/jp512153b (accessed Feb 26, 2018).

(23) Gao, W.; Kahn, A. Electronic Structure and Current Injection in Zinc Phthalocyanine Doped with Tetrafluorotetracyanoquinodimethane: Interface versus Bulk Effects. Org. Electron. 2002, 3 (2), 53-63.

(24) Perdew, J. P.; Burke, K.; Ernzerhof, M. Generalized Gradient Approximation Made Simple. Phys. Rev. Lett. 1996, 77 (18), 3865-3868.

(25) Perdew, J. P.; Burke, K.; Ernzerhof, M. ERRATA: Generalized Gradient Approximation Made Simple [Phys. Rev. Lett. 77, 3865 (1996)]. Phys. Rev. Lett. 1997, 78 (7), 1396-1396.

(26) Zhang, Y.; Yang, W. A Challenge for Density Functionals: Self-Interaction Error Increases for Systems with a Noninteger Number of Electrons. J. Chem. Phys. 1998, 109 (7), 2604-2608.

(27) Mori-Sánchez, P.; Cohen, A. J.; Yang, W. Many-Electron Self-Interaction Error in Approximate Density Functionals. J. Chem. Phys. 2006, 125 (20), 201102.

(28) Kümmel, S.; Kronik, L. Orbital-Dependent Density Functionals: Theory and Applications. Rev. Mod. Phys. 2008, 80 (1), 3-60.

(29) Perdew, J. P.; Ruzsinszky, A.; Constantin, L. A.; Sun, J.; Csonka, G. I. Some Fundamental Issues in Ground-State Density Functional Theory: A Guide for the Perplexed. J. Chem. Theory Comput. 2009, 5 (4), 902-908.

(30) Perdew, J. P.; Ernzerhof, M.; Burke, K. Rationale for Mixing Exact Exchange with Density Functional Approximations. J. Chem. Phys. 1996, 105 (22), 9982-9985.

(31) Ivády, V.; Armiento, R.; Szász, K.; Janzén, E.; Gali, A.; Abrikosov, I. A. Theoretical Unification of Hybrid-DFT and DFT + U Methods for the Treatment of Localized Orbitals. Phys. Rev. B 2014, $90(3), 035146$.

(32) Verma, P.; Truhlar, D. G. Does DFT+U Mimic Hybrid Density Functionals? Theor. Chem. Acc. 2016, $135(8), 182$.

(33) Gani, T. Z. H.; Kulik, H. J. Where Does the Density Localize? Convergent Behavior for Global Hybrids, Range Separation, and DFT+U. J. Chem. Theory Comput. 2016, 12 (12), 5931-5945.

(34) Hofmann, D.; Kümmel, S. Integer Particle Preference during Charge Transfer in Kohn-Sham Theory. Phys. Rev. B 2012, 86 (20), 201109.

(35) Adamo, C.; Barone, V. Toward Reliable Density Functional Methods without Adjustable Parameters: The PBEO Model. J. Chem. Phys. 1999, 110 (13), 6158-6170.

(36) Blum, V.; Gehrke, R.; Hanke, F.; Havu, P.; Havu, V.; Ren, X.; Reuter, K.; Scheffler, M. Ab Initio Molecular Simulations with Numeric Atom-Centered Orbitals. Comput. Phys. Commun. 2009, 180 (11), 2175-2196.

(37) Tkatchenko, A.; Scheffler, M. Accurate Molecular Van Der Waals Interactions from GroundState Electron Density and Free-Atom Reference Data. Phys. Rev. Lett. 2009, 102 (7), 073005.

(38) Ruiz, V. G.; Liu, W.; Zojer, E.; Scheffler, M.; Tkatchenko, A. Density-Functional Theory with Screened van Der Waals Interactions for the Modeling of Hybrid Inorganic-Organic Systems. Phys. Rev. Lett. 2012, 108 (14), 146103.

(39) Soon, A.; Todorova, M.; Delley, B.; Stampfl, C. Thermodynamic Stability and Structure of Copper Oxide Surfaces: A First-Principles Investigation. Phys. Rev. B 2007, 75 (12), 125420.

(40) Önsten, A.; Göthelid, M.; Karlsson, U. O. Atomic Structure of Cu2O(1 11 1). Surf. Sci. 2009, 603 (2), 257-264. 
(41) Xu, Y.; Hofmann, O. T.; Schlesinger, R.; Winkler, S.; Frisch, J.; Niederhausen, J.; Vollmer, A.; Blumstengel, S.; Henneberger, F.; Koch, N.; et al. Space-Charge Transfer in Hybrid InorganicOrganic Systems. Phys. Rev. Lett. 2013, 111 (22), 226802.

(42) Hofmann, O. T.; Deinert, J.-C.; Xu, Y.; Rinke, P.; Stähler, J.; Wolf, M.; Scheffler, M. Large Work Function Reduction by Adsorption of a Molecule with a Negative Electron Affinity: Pyridine on ZnO(101º). J. Chem. Phys. 2013, 139 (17), 174701.

(43) Meyer, B. K.; Polity, A.; Reppin, D.; Becker, M.; Hering, P.; Klar, P. J.; Sander, T.; Reindl, C.; Benz, J.; Eickhoff, M.; et al. Binary Copper Oxide Semiconductors: From Materials towards Devices. Phys. Status Solidi B 2012, 249 (8), 1487-1509.

(44) Neugebauer, J.; Scheffler, M. Adsorbate-Substrate and Adsorbate-Adsorbate Interactions of $\mathrm{Na}$ and K Adlayers on Al(111). Phys. Rev. B 1992, 46 (24), 16067-16080.

(45) Anderson, P. W. Localized Magnetic States in Metals. Phys. Rev. 1961, 124 (1), 41-53.

(46) NEWNS, D. M. Self-Consistent Model of Hydrogen Chemisorption. Phys. Rev. 1969, 178 (3), 1123-1135.

(47) Lindell, L.; Unge, M.; Osikowicz, W.; Stafström, S.; Salaneck, W. R.; Crispin, X.; de Jong, M. P. Integer Charge Transfer at the Tetrakis(Dimethylamino)Ethylene/Au Interface. Appl. Phys. Lett. 2008, 92 (16), 163302.

(48) Murdey, R. J.; Salaneck, W. R. Charge Injection Barrier Heights Across Multilayer Organic Thin Films. Jpn. J. Appl. Phys. 2005, 44 (6R), 3751.

(49) Braun, S.; Salaneck, W. R. Fermi Level Pinning at Interfaces with Tetrafluorotetracyanoquinodimethane (F4-TCNQ): The Role of Integer Charge Transfer States. Chem. Phys. Lett. 2007, 438 (4-6), 259-262.

(50) Swart, I.; Sonnleitner, T.; Repp, J. Charge State Control of Molecules Reveals Modification of the Tunneling Barrier with Intramolecular Contrast. Nano Lett. 2011, 11 (4), 1580-1584.

(51) Png, R.-Q.; Ang, M. C. Y.; Teo, M.-H.; Choo, K.-K.; Tang, C. G.; Belaineh, D.; Chua, L.-L.; Ho, P. K. $\mathrm{H}$. Madelung and Hubbard Interactions in Polaron Band Model of Doped Organic Semiconductors. Nat. Commun. 2016, 7, 11948.

(52) Mermin, N. D. Thermal Properties of the Inhomogeneous Electron Gas. Phys. Rev. 1965, 137 (5A), A1441-A1443.

(53) Perdew, J. P.; Parr, R. G.; Levy, M.; Balduz, J. L. Density-Functional Theory for Fractional Particle Number: Derivative Discontinuities of the Energy. Phys. Rev. Lett. 1982, 49 (23), 1691-1694.

(54) Stein, T.; Autschbach, J.; Govind, N.; Kronik, L.; Baer, R. Curvature and Frontier Orbital Energies in Density Functional Theory. J. Phys. Chem. Lett. 2012, 3 (24), 3740-3744.

(55) Kraisler, E.; Kronik, L. Piecewise Linearity of Approximate Density Functionals Revisited: Implications for Frontier Orbital Energies. Phys. Rev. Lett. 2013, 110 (12), 126403.

(56) Perdew, J. P.; Ruzsinszky, A.; Csonka, G. I.; Vydrov, O. A.; Scuseria, G. E.; Staroverov, V. N.; Tao, J. Exchange and Correlation in Open Systems of Fluctuating Electron Number. Phys. Rev. A 2007, 76 (4), 040501.

(57) Vydrov, O. A.; Scuseria, G. E.; Perdew, J. P. Tests of Functionals for Systems with Fractional Electron Number. J. Chem. Phys. 2007, 126 (15), 154109.

(58) Körzdörfer, T.; Brédas, J.-L. Organic Electronic Materials: Recent Advances in the DFT Description of the Ground and Excited States Using Tuned Range-Separated Hybrid Functionals. Acc. Chem. Res. 2014, 47 (11), 3284-3291.

(59) Janak, J. F. Proof That $\partial E / \partial n i=\varepsilon$ in Density-Functional Theory. Phys. Rev. B 1978, 18 (12), 7165-7168.

(60) Cohen, A. J.; Mori-Sánchez, P.; Yang, W. Development of Exchange-Correlation Functionals with Minimal Many-Electron Self-Interaction Error. J. Chem. Phys. 2007, 126 (19), 191109.

(61) Heaton-Burgess, T.; Yang, W. Structural Manifestation of the Delocalization Error of Density Functional Approximations: $\mathrm{C} 4 \mathrm{~N}+2$ Rings and C20 Bowl, Cage, and Ring Isomers. J. Chem. Phys. 2010, 132 (23), 234113. 
(62) Heyd, J.; Scuseria, G. E.; Ernzerhof, M. Hybrid Functionals Based on a Screened Coulomb Potential. J. Chem. Phys. 2003, 118 (18), 8207-8215.

(63) Heyd, J.; Scuseria, G. E.; Ernzerhof, M. Erratum: "Hybrid Functionals Based on a Screened Coulomb Potential" [J. Chem. Phys.118, 8207 (2003)]. J. Chem. Phys. 2006, 124 (21), 219906.

(64) Stein, T.; Eisenberg, H.; Kronik, L.; Baer, R. Fundamental Gaps in Finite Systems from Eigenvalues of a Generalized Kohn-Sham Method. Phys. Rev. Lett. 2010, 105 (26), 266802.

(65) Refaely-Abramson, S.; Baer, R.; Kronik, L. Fundamental and Excitation Gaps in Molecules of Relevance for Organic Photovoltaics from an Optimally Tuned Range-Separated Hybrid Functional. Phys. Rev. B 2011, 84 (7), 075144.

(66) Kronik, L.; Stein, T.; Refaely-Abramson, S.; Baer, R. Excitation Gaps of Finite-Sized Systems from Optimally Tuned Range-Separated Hybrid Functionals. J. Chem. Theory Comput. 2012, 8 (5), 1515-1531.

(67) Karolewski, A.; Kronik, L.; Kümmel, S. Using Optimally Tuned Range Separated Hybrid Functionals in Ground-State Calculations: Consequences and Caveats. J. Chem. Phys. 2013, 138 (20), 204115.

(68) Gledhill, J. D.; Peach, M. J. G.; Tozer, D. J. Assessment of Tuning Methods for Enforcing Approximate Energy Linearity in Range-Separated Hybrid Functionals. J. Chem. Theory Comput. 2013, 9 (10), 4414-4420.

(69) Sun, H.; Autschbach, J. Influence of the Delocalization Error and Applicability of Optimal Functional Tuning in Density Functional Calculations of Nonlinear Optical Properties of Organic Donor-Acceptor Chromophores. ChemPhysChem 2013, 14 (11), 2450-2461.

(70) Hofmann, O. T.; Atalla, V.; Moll, N.; Rinke, P.; Scheffler, M. Interface Dipoles of Organic Molecules on $\mathrm{Ag}(111)$ in Hybrid Density-Functional Theory. New J. Phys. 2013, 15 (12), 123028.

(71) Sai, N.; Barbara, P. F.; Leung, K. Hole Localization in Molecular Crystals from Hybrid Density Functional Theory. Phys. Rev. Lett. 2011, 106 (22), 226403.

(72) Yang, W.; Cohen, A. J.; Mori-Sánchez, P. Derivative Discontinuity, Bandgap and Lowest Unoccupied Molecular Orbital in Density Functional Theory. J. Chem. Phys. 2012, 136 (20), 204111.

(73) Atalla, V.; Yoon, M.; Caruso, F.; Rinke, P.; Scheffler, M. Hybrid Density Functional Theory Meets Quasiparticle Calculations: A Consistent Electronic Structure Approach. Phys. Rev. B 2013, 88 (16), 165122.

(74) Perdew, J. P.; Levy, M. Physical Content of the Exact Kohn-Sham Orbital Energies: Band Gaps and Derivative Discontinuities. Phys. Rev. Lett. 1983, 51 (20), 1884-1887.

(75) Sham, L. J.; Schlüter, M. Density-Functional Theory of the Energy Gap. Phys. Rev. Lett. 1983, 51 (20), 1888-1891.

(76) Gould, T.; Toulouse, J. Kohn-Sham Potentials in Exact Density-Functional Theory at Noninteger Electron Numbers. Phys. Rev. A 2014, 90 (5), 050502.

(77) Rios-Font, R.; Sodupe, M.; Rodríguez-Santiago, L.; Taylor, P. R. The Role of Exact Exchange in the Description of Cu2+-(H2O)n $(\mathrm{n}=1-6)$ Complexes by Means of DFT Methods. J. Phys. Chem. A 2010, 114 (40), 10857-10863.

(78) Gunnarsson, O.; Lundqvist, B. I. Exchange and Correlation in Atoms, Molecules, and Solids by the Spin-Density-Functional Formalism. Phys. Rev. B 1976, 13 (10), 4274-4298.

(79) Vydrov, O. A.; Scuseria, G. E. Ionization Potentials and Electron Affinities in the PerdewZunger Self-Interaction Corrected Density-Functional Theory. J. Chem. Phys. 2005, 122 (18), 184107.

(80) Rostgaard, C.; Jacobsen, K. W.; Thygesen, K. S. Fully Self-Consistent GW Calculations for Molecules. Phys. Rev. B 2010, 81 (8), 085103.

(81) Neaton, J. B.; Hybertsen, M. S.; Louie, S. G. Renormalization of Molecular Electronic Levels at Metal-Molecule Interfaces. Phys. Rev. Lett. 2006, 97 (21), 216405. 
(82) Garcia-Lastra, J. M.; Rostgaard, C.; Rubio, A.; Thygesen, K. S. Polarization-Induced Renormalization of Molecular Levels at Metallic and Semiconducting Surfaces. Phys. Rev. B 2009, 80 (24), 245427.

(83) Thygesen, K. S.; Rubio, A. Renormalization of Molecular Quasiparticle Levels at MetalMolecule Interfaces: Trends across Binding Regimes. Phys. Rev. Lett. 2009, 102 (4), 046802.

(84) Biller, A.; Tamblyn, I.; Neaton, J. B.; Kronik, L. Electronic Level Alignment at a Metal-Molecule Interface from a Short-Range Hybrid Functional. J. Chem. Phys. 2011, 135 (16), 164706.

(85) Rinke, P.; Qteish, A.; Neugebauer, J.; Scheffler, M. Exciting Prospects for Solids: ExactExchange Based Functionals Meet Quasiparticle Energy Calculations. Phys Stat Sol B 2008, 245 (5), 929-945.

(86) Blase, X.; Attaccalite, C.; Olevano, V. First-Principles GW Calculations for Fullerenes, Porphyrins, Phtalocyanine, and Other Molecules of Interest for Organic Photovoltaic Applications. Phys. Rev. B 2011, 83 (11), 115103.

(87) Sinai, O.; Hofmann, O. T.; Rinke, P.; Scheffler, M.; Heimel, G.; Kronik, L. Multiscale Approach to the Electronic Structure of Doped Semiconductor Surfaces. Phys. Rev. B 2015, 91 (7), 075311.

(88) Erker, S.; Rinke, P.; Moll, N.; Hofmann, O. T. Doping Dependence of the Surface Phase Stability of Polar O-Terminated (000I) ZnO. New J. Phys. 2017, 19 (8), 083012.

(89) Yang, J.-P.; Bussolotti, F.; Kera, S.; Ueno, N. Origin and Role of Gap States in Organic Semiconductor Studied by UPS: As the Nature of Organic Molecular Crystals. J. Phys. Appl. Phys. 2017, 50 (42), 423002.

(90) Winkler, S.; Amsalem, P.; Frisch, J.; Oehzelt, M.; Heimel, G.; Koch, N. Probing the Energy Levels in Hole-Doped Molecular Semiconductors. Mater. Horiz. 2015, 2 (4), 427-433.

(91) Mulliken, R. S. Electronic Population Analysis on LCAO-MO Molecular Wave Functions. I. J. Chem. Phys. 1955, 23 (10), 1833-1840.

(92) Hirshfeld, F. L. Bonded-Atom Fragments for Describing Molecular Charge Densities. Theor. Chim. Acta 1977, 44 (2), 129-138.

(93) Rangger, G. M.; Hofmann, O. T.; Romaner, L.; Heimel, G.; Bröker, B.; Blum, R.-P.; Johnson, R. L.; Koch, N.; Zojer, E. F4TCNQ on $\mathrm{Cu}, \mathrm{Ag}$, and Au as Prototypical Example for a Strong Organic Acceptor on Coinage Metals. Phys. Rev. B 2009, 79 (16), 165306. 


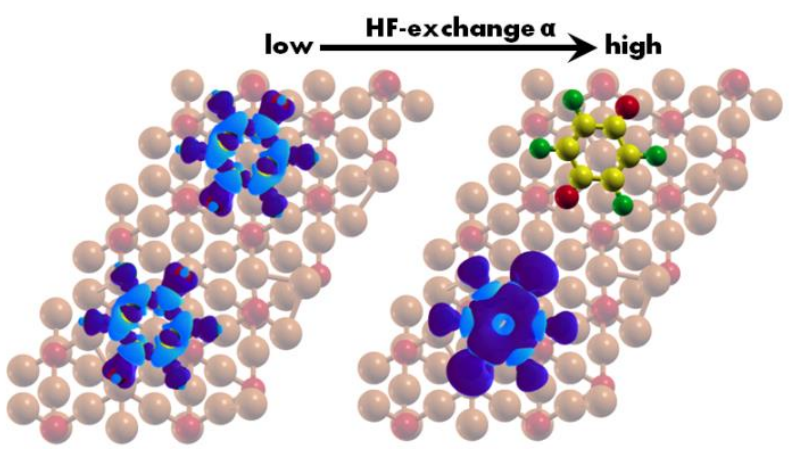

